Old Dominion University

ODU Digital Commons

Time Domain Boundary Element Method Prediction of Noise Shielding by a NACA 0012 Airfoil

Douglas M. Nark

Fang Q. Hu

Old Dominion University, fhu@odu.edu

Follow this and additional works at: https://digitalcommons.odu.edu/mathstat_fac_pubs

Part of the Aerodynamics and Fluid Mechanics Commons, Aeronautical Vehicles Commons, and the Transportation Commons

Original Publication Citation

Nark, D. M., \& Hu, F. Q. (2021). Time domain boundary element method prediction of noise shielding by a NACA 0012 airfoil. AIAA Aviation 2021 Forum, August 2-6, 2021, Virtual. https://doi.org/10.2514/ $6.2021-2201$

This Conference Paper is brought to you for free and open access by the Mathematics \& Statistics at ODU Digital Commons. It has been accepted for inclusion in Mathematics \& Statistics Faculty Publications by an authorized administrator of ODU Digital Commons. For more information, please contact digitalcommons@odu.edu. 


\title{
Time Domain Boundary Element Method Prediction of Noise Shielding by an NACA 0012 Airfoil
}

\author{
Douglas M. Nark* \\ NASA Langley Research Center, Hampton, VA 23681-2199, U.S.A \\ Fang Q. $\mathrm{Hu}^{\dagger}$ \\ Old Dominion University, Norfolk, VA 23529, USA
}

\begin{abstract}
As aircraft noise constraints become more stringent and the number/mixture of aircraft configurations grows, it becomes more important to understand the interaction of individual aircraft noise sources with nearby aircraft structures. Understanding these interactions and exploring possible approaches to mitigate or exploit their acoustic impact is essential for overcoming key noise barriers. This paper describes the further validation of a time domain boundary element approach for the prediction of the interactions between incident noise sources and nearby aircraft structures. Predictions were completed for multiple source locations and comparisons of these results with measured data are presented. Overall, very good agreement between the predicted and measured quantities was obtained in both the pressure time histories and pressure spectra. The effects of surface mesh resolution and source waveform are also presented. The very promising results demonstrate the capabilities of the time domain methodology employed in this study and provide further confidence in its continued development and application in future studies.
\end{abstract}

\section{Introduction}

As aircraft noise constraints become more stringent and the number/mixture of aircraft configurations grows, the need for improved aircraft noise reduction technologies becomes more evident. To achieve more aggressive noise reduction goals, it becomes more important to not only understand individual aircraft noise sources, but their interaction with nearby aircraft structures as well. For example, the mutual interaction of multiple propulsors and the airframe structure can lead to increased system noise levels. However, these interactions may also offer opportunities for noise reduction. Understanding these interactions and exploring possible approaches to mitigate or exploit their acoustic impact is important for overcoming key noise barriers.

This paper presents further validation of a time domain approach for the prediction of the interactions between incident noise sources and nearby aircraft structures. Specifically, predictions using a time domain boundary element method ${ }^{122}$ are compared with measured data from a fundamental shielding experiment ${ }^{3 / 4}$ The test facility and associated measurements are described in Section [I] After a brief discussion of the formulation in Section [III, the computational model is then presented in Section IV Comparisons of the predictions with measured data are provided in Section VI Effects of computational mesh, side edge scattering, and source modeling are discussed in Sections VII IX] Finally, concluding remarks regarding some of the more significant results and further areas of interest are presented in Section $\mathrm{X}$

\section{Test Facility and Acoustic Measurements}

The measured data used in this study were obtained from experiments conducted in the NASA Langley Quiet Flow Facility (QFF). While full details of the experiments are discussed in previous papers,$\frac{314}{}$ background information on the test setup and measurement locations provides context for discussion of the prediction approach and subsequent

\footnotetext{
*Senior Research Scientist, Structural Acoustics Branch, Research Directorate, AIAA Associate Fellow

${ }^{\dagger}$ Professor, Department of Mathematics and Statistics, AIAA Associate Fellow
} 
data comparisons. The QFF is an anechoic facility equipped with a 0.61 by $0.91 \mathrm{~m}$ rectangular open jet nozzle. The acoustic shielding/scattering model model was a wing with an NACA 0012 airfoil having a $0.254 \mathrm{~mm}$ thick trailing edge, a $0.91 \mathrm{~m}$ span, and a $0.20 \mathrm{~m}$ chord. As seen in Figure 1. the model was positioned at the center of the test section and a traversing microphone was used to acquire the acoustic measurements inside the test section at different streamwise stations. The sound source was a laser-induced plasma that generated a nearly omnidirectional pressure wave that propagated as an isentropic acoustic wave in the far field.

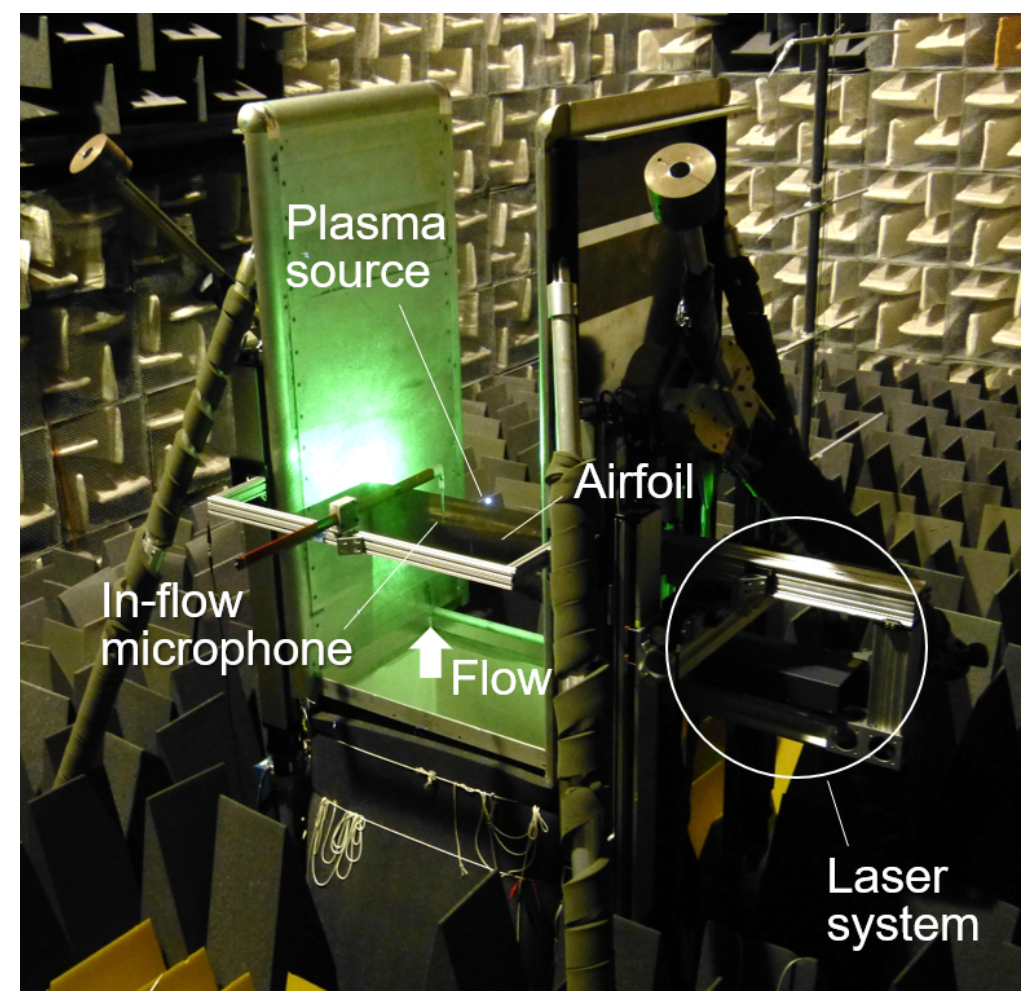

Figure 1: Quiet Flow Facility (QFF) experimental setup $\frac{3}{3}$

Each set of in-flow microphone surveys was acquired with and without the model installed to produce shielded and unshielded configurations. Measurements were acquired in the midspan plane of the model at distances of one chord $(200 \mathrm{~mm})$ and two chords $(400 \mathrm{~mm})$ from the test section centerline. Measurements performed two chords away from the test section centerline placed the microphone outside of the test section, and were therefore only performed without flow. The various sound source (plasma) and microphone survey locations are shown in Figure 2 The survey microphone was traversed in the streamwise direction, up to $200 \mathrm{~mm}$ upstream and $400 \mathrm{~mm}$ downstream of the midspan airfoil section leading edge. In Figure 2, the open black circles indicate microphone locations where measurements were acquired for most test configurations, while the smaller closed circles indicate measurement locations used for only a subset of test cases. Sound source positions of 0\% (airfoil leading edge), 25\%, 50\%, 70\%, 75\% and 100\% chord are included as solid green circles. For each of these locations, the source was positioned at a constant normal distance of $25 \mathrm{~mm}$ from the airfoil surface. For the axial source position of $70 \%$ chord, additional measurements were acquired with the source at a normal distance of $40 \mathrm{~mm}$ from the airfoil surface.

\section{Mathematical Formulation}

Under a uniform mean flow assumption, propagation and scattering of acoustic pressure of small amplitudes can be modeled by the linear convective wave equation as:

$$
\left(\frac{\partial}{\partial t}+U \cdot \nabla\right)^{2} p-a^{2} \nabla^{2} p=s(r, t)
$$




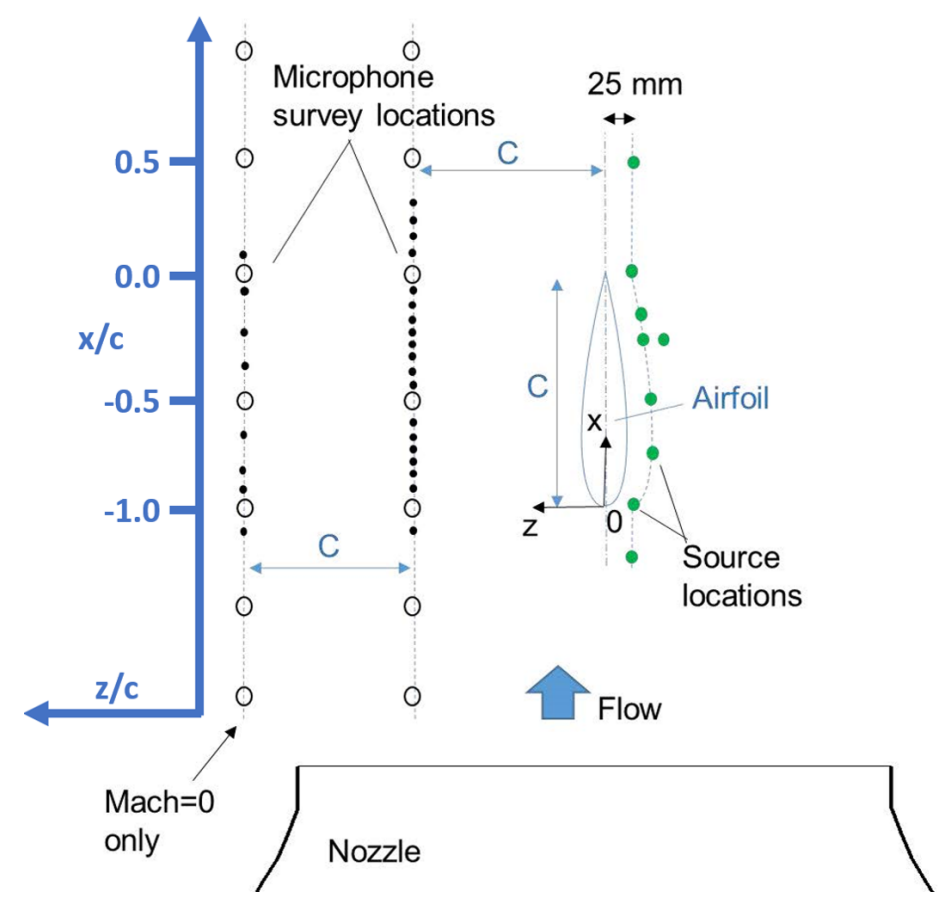

Figure 2: QFF plasma source and microphone locations (Note: $c=200 \mathrm{~mm}$ ).

where $p(r, t)$ is the acoustic pressure, $U$ is the uniform mean flow, $a$ is the speed of sound, and $s(r, t)$ is the source term. Also, $\nabla=\left(\frac{\partial}{\partial x}, \frac{\partial}{\partial y}, \frac{\partial}{\partial z}\right)$ and $\nabla^{2}=\nabla \cdot \nabla$. In addition, on the surface of airfoil $S$, the Zero Energy Flux (ZEF) solid wall boundary condition is assumed for the pressure $p$ :

$$
\frac{\partial p}{\partial \tilde{n}}=0, \text { on } S
$$

where the combined normal derivative operator $\frac{\partial}{\partial \tilde{n}}$ is defined as follows:

$$
\frac{\partial}{\partial \tilde{n}}=\frac{\partial}{\partial \bar{n}}-\frac{M_{n}}{a} \frac{\partial}{\partial t}, \frac{\partial}{\partial \bar{n}}=\frac{\partial}{\partial n}-M_{n}(M \cdot \nabla), \quad M=\frac{U}{a}, \quad M_{n}=M \cdot n
$$

in which $n$ is the surface normal vector pointing into the solid body. As described by Hu et al. ${ }^{[5]}$ the ZEF condition (Eq. 2) is the same as the usual pressure boundary condition on solid surfaces (i.e., its normal derivative being zero) in the absence of a mean flow $(M=0)$. However, in the presence of a nonzero mean flow, the ZEF condition ensures conservation of the energy quantity $E$ associated with the convective wave equation (Eq. 1) where

$$
E=\frac{1}{2}|\nabla p|^{2}+\frac{1}{2 a^{2}}\left|\frac{\partial p}{\partial t}+U \cdot \nabla p\right|^{2}-\frac{U \cdot \nabla p}{a^{2}}\left(\frac{\partial p}{\partial t}+U \cdot \nabla p\right) .
$$

With rigid wall boundary condition (Eq.22, the convective wave equation (Eq.11) can be converted into the following time domain boundary integral equation:

$$
4 \pi C_{s} p\left(r^{\prime}, t^{\prime}\right)+\int_{S} \frac{\partial G_{0}}{\partial \bar{n}}\left(p\left(r_{s}, t_{R}^{\prime}\right)+\frac{\bar{R}}{a \alpha^{2}} \frac{\partial p}{\partial t}\left(r_{s}, t_{R}^{\prime}\right)\right) d r_{s}=\frac{1}{a^{2}} \int_{V_{s}} \frac{1}{\bar{R}} s\left(r, t_{R}^{\prime}\right) d r
$$

where $C_{s}=1 / 2$ when $r^{\prime}$ is a surface point and $C_{s}=1$ when $r^{\prime}$ is an off-surface field point. In the above,

$$
G_{0}=\frac{1}{\bar{R}\left(r, r^{\prime}\right)}, \bar{R}\left(r, r^{\prime}\right)=\sqrt{\left[M \cdot\left(r-r^{\prime}\right)\right]^{2}+\alpha^{2}\left|r-r^{\prime}\right|^{2}}, t_{R}^{\prime}=t^{\prime}+\beta \cdot\left(r^{\prime}-r\right)-\frac{\bar{R}}{a \alpha^{2}}
$$

in which 


$$
\alpha=\sqrt{1-|M|^{2}}, \beta=\frac{M}{a \alpha^{2}} .
$$

In Equation 5, $V_{s}$ denotes the volume where source term $s(r, t)$ is nonzero. To facilitate the comparison with experimental measurements in the NASA QFF facility, the source function $s(r, t)$ in Eq. 5 is taken to be a point source of the form:

$$
s(r, t)=4 \pi a^{2} q_{Q F F}(t) \delta\left(r-r_{q}\right)
$$

where $r_{q}$ denotes coordinates for the source point, and $q_{Q F F}(t)$ is the source waveform, which resembles that found in the QFF experiment.

\section{Computational Modeling}

Equation 5 is solved by a time domain boundary element method detailed in previous studies $1 / 6$ A schematic diagram for the setup of the computational domain is shown in Figure 3 . The coordinate system is such that $x$ is in the streamwise direction and $y$ in the wing spanwise direction. At zero degrees angle of attack, the chord of the midspan airfoil section is located from the leading edge coordinates $(x, y, z)=(-c, 0,0)$ to the trailing edge $(x, y, z)=(0,0,0)$, where $c$ is the chord length (see Fig. 2). In addition, a set of off-surface field points are included in the computation where time histories of the solution are recorded. In particular, 1201 field points are included along each of the field lines placed at $(x, 0, c)$ and $(x, 0,2 c),-3 \leq x / c \leq 3$, which coincide with the lines of microphones in the QFF facility $[3$

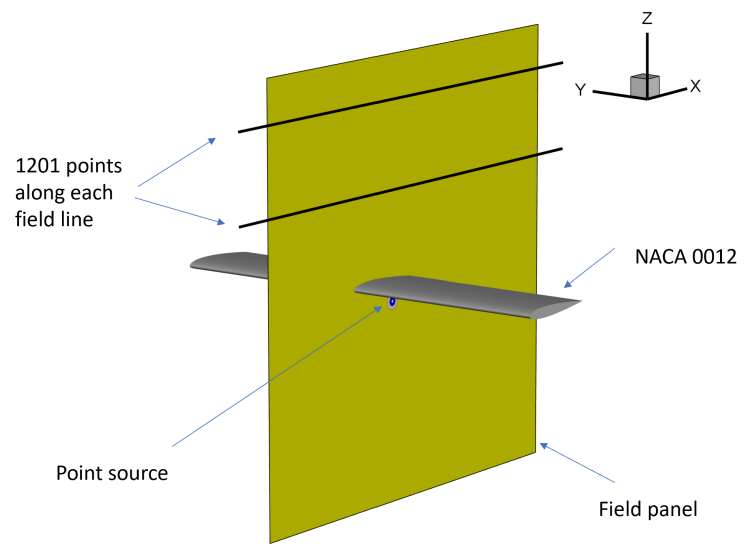

Figure 3: Computational domain for time domain boundary element method.

In a boundary element method, only a surface mesh is needed for the solution of the integral equation. For most of the computational results presented here, the upper and lower surfaces of the wing are discretized by rectangular elements. On side surfaces of the wing, unstructured elements are used due to the shape of the airfoil. For convenience of applying high-order numerical quadrature for surface integration, any triangular elements are divided into three quadrilateral elements. To resolve the sharp trailing edge of the NACA0012 airfoil, elements are progressively refined toward the trailing edge, while maintaining a limit on the maximum aspect ratio of the elements. Three meshes of varying resolution, referred to as Mesh \#1, \#2 and \#3, respectively, have been constructed to study the convergence of the numerical solutions. The total number of elements and other statistics of the meshes are detailed in Table 1 Visualizations of the three meshes are given in Figure 4. The proper element sizes on the surface of the wing are determined using the following rule:

$$
\ell \leq \frac{1}{4}\left(\frac{a}{f_{\max }}\right)
$$

where $\ell$ is the allowable side length of a surface element, $f_{\max }$ is the maximum frequency to be resolved and $a$ is the speed of sound. This results in at least 4 elements per wave length, or 16 points-per-wavelength-squared $\left(\mathrm{PPW}^{2}\right) \mathbf{7}^{7}$ Meshes \#1, \#2, and \#3 are designed to resolve frequencies up to $40 \mathrm{kHz}, 80 \mathrm{kHz}$, and $100 \mathrm{kHz}$, respectively. 


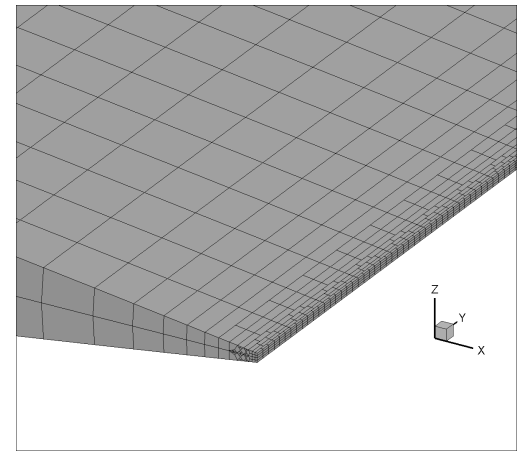

(a) Mesh \#1 $\left(f_{\max }=40 \mathrm{kHz}\right)$

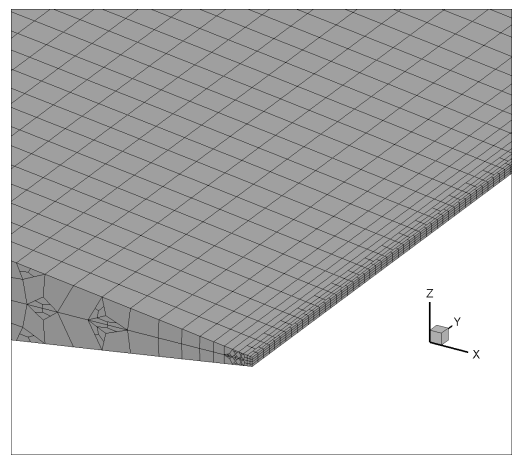

(b) Mesh \#2 $\left(f_{\max }=80 \mathrm{kHz}\right)$

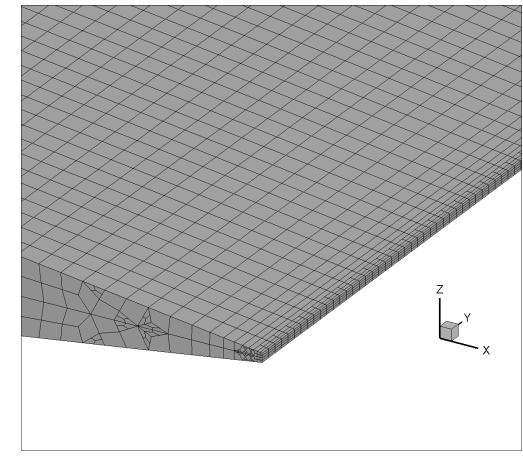

(c) Mesh \#3 $\left(f_{\text {max }}=100 \mathrm{kHz}\right)$

Figure 4: Portions of the surface meshes for Mesh \#1, \#2, and \#3, showing the variation in spatial resolution and the progressive refinement toward the trailing edge.

Table 1: Surface mesh information for Mesh \#1,\#2, and \#3. Element length is scaled by the chord length $c$.

\begin{tabular}{|c|c|c|c|}
\hline & Mesh \#1 & Mesh \#2 & Mesh \#3 \\
\hline \hline Number of elements & 183,700 & 590,724 & 965,656 \\
\hline Max element length & 0.01 & 0.005 & 0.004 \\
\hline Min element length & 0.000134 & 0.000134 & 0.000134 \\
\hline Max element aspect ratio & 5 & 5 & 5 \\
\hline Expected frequency resolution & $40 \mathrm{kHz}$ & $80 \mathrm{kHz}$ & $100 \mathrm{kHz}$ \\
\hline
\end{tabular}

\section{Modeling of Source Waveform}

As mentioned earlier, acoustic pressure is computed in this work by solving the convective wave equation for pressure with a source term. In order to compare the computational solution with the experimental measurements in the time domain, the computational source term should mimic the actual QFF incident pressure waveform ${ }^{3}$ as closely as possible. However, comparison of integrated shielding metrics (like the shielding factor described in Section VI) may not be as dependent on the specific computational source waveform.

In the QFF experimental study, 3 acoustic pressures without the model installed (i.e., in an unshielded configuration) were measured at eight microphone locations (see Fig. 2p. These measurements provide the acoustic pressure waveform of the incident point source employed in the QFF experiments. As mentioned above, mathematical modeling of the source term in the wave equation (Eq. 1) should be such that the incident waveform is reproduced in the computation. Figure 5a shows the measured pressure signal, $p_{i}$, at the eight microphone locations for the case where the source point is placed at 75\% chord with coordinates $r_{q}=(-0.25 c, 0,-0.157 c)$. The microphone locations are as indicated in the figure legend. The pressure values (scaled by $d / c$ ) are plotted as a function of $t-d / a$ where $d=\left|r_{o}-r_{q}\right|$ denotes the distance between the observer point $r_{o}$ and the source point $r_{q}, c$ is the chord length, and $a$ is the speed of sound. It is seen that pressure waveform consists of a main pulse formed by the first peak and trough, followed by weaker secondary waves. As expected, when the acoustic pressure at the observer point is scaled by its distance from the source and the observer time is adjusted by the retarded time, the measured pressure waveforms show a high degree of similarity. However, there are also some small deviations in the measured pressure peak amplitude and arrival time. On the other hand, when the pressure time histories are normalized by their peak value, $p_{0}$, and the arrival time, $t_{0}$, for each microphone location (see Fig. 5b), very good convergence for the measured incident waveforms is observed (particularly for the main peak and trough). However, there are noticeable variations among the eight locations for the weaker secondary waves. These variations may be attributed to small distortions that are location dependent, such as atmospheric attenuation, nonlinear propagation effects, and microphone responses $\frac{8}{8}$ The effects of these deviations in the secondary waves on the computational solutions will be discussed further in subsequent sections. 
For computational purposes, the experimental waveform measured at one of the eight locations, specifically at $r_{o}=(-1.5 c, 0, c)$, is selected for the simulation. Therefore, the source time function $q_{Q F F}(t)$ in Eq. 6 is determined so that the direct unshielded pressure from the point source at $r_{q}$ matches the measurement at $r_{o}$. That is, we let

$$
\frac{1}{d} q_{Q F F}(t)=p_{i}\left(t-t_{0}\right)
$$

where $d=\left|r_{o}-r_{q}\right|$ for the case of no flow and $t_{0}$ is the first peak arrival time for the measured pressure, $p_{i}(t)$. Note that in Eq. 8, $t$ is the source time and the first peak pressure of the point source is emitted at $t=0$.

For convenience of computation, the experimental waveform is modeled using multiple analytical Gaussian functions, which are smooth and infinitely differentiable. Specifically, the source time function $q_{Q F F}(t)$ appearing in Eq.6 is given as:

$$
\begin{gathered}
q_{Q F F}(t)=c\left[491.71 e^{-30426(t-0.00035)^{2}}-367.71 e^{-14862(t-0.01291)^{2}}+40.79 e^{-70580(t-0.02981)^{2}}\right. \\
\left.-11.57 e^{-89104(t-0.03885)^{2}}+16.95 e^{-54353(t-0.04813)^{2}}-11.84 e^{-43456(t-0.05836)^{2}}+5.86 e^{-60472(t-0.06931)^{2}}\right]
\end{gathered}
$$

where $t$ is source time in milliseconds and $c$ is the chord length. Each of the Gaussian functions approximately represents a peak/trough in the waveform and the coefficients are found using a Python differential evolution optimization routine. Comparison of the time history and spectrum for the analytical model and measured data are shown in Figures $6 \mathrm{a}$ and $6 \mathrm{~b}$. Both are in very good agreement.

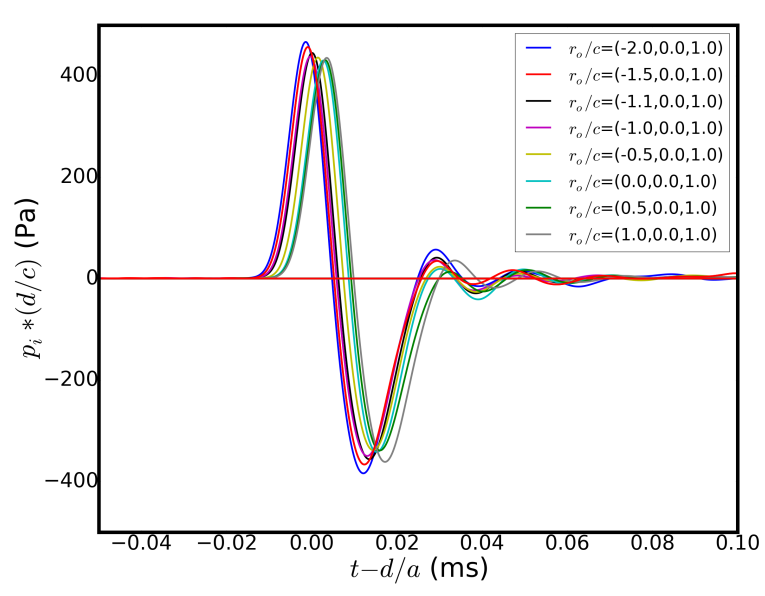

(a)

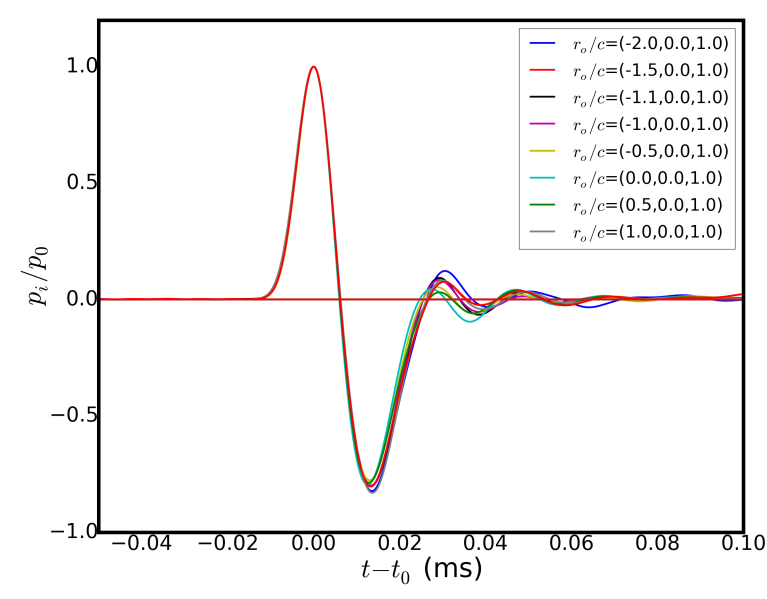

(b)

Figure 5: Measured unshielded pressure signal $p_{i}$ at the eight far field microphone locations. The point source is located at $r_{q}=(-0.25 c, 0,157 c)$ and the microphone locations $r_{o}$ are as indicated. (a) $p_{i} \times(d / c)$ vs. time $t$ with the retarded time subtracted, where $d$ is the distance from source point, $r_{q}$, to the observer point, $r_{o}$, and $c$ is the chord length; (b) Pressure, $p_{i}$, normalized by its peak value, denoted as $p_{0}$, at time $t_{0}$.

\section{Computational results and comparison with experimental measurements}

In this section, computational results for three source locations are presented and compared with the experimental measurements. In the first two cases, where the point source is located at 75\% chord (Case 75c) and the leading edge (Case LE), respectively, direct comparison of both the time domain and frequency domain results are presented. For the third case, the point source is placed at 70\% chord (Case 70c). This is a benchmark case that has been studied by multiple research groups and only frequency domain comparisons will be presented (with and without mean flow). 


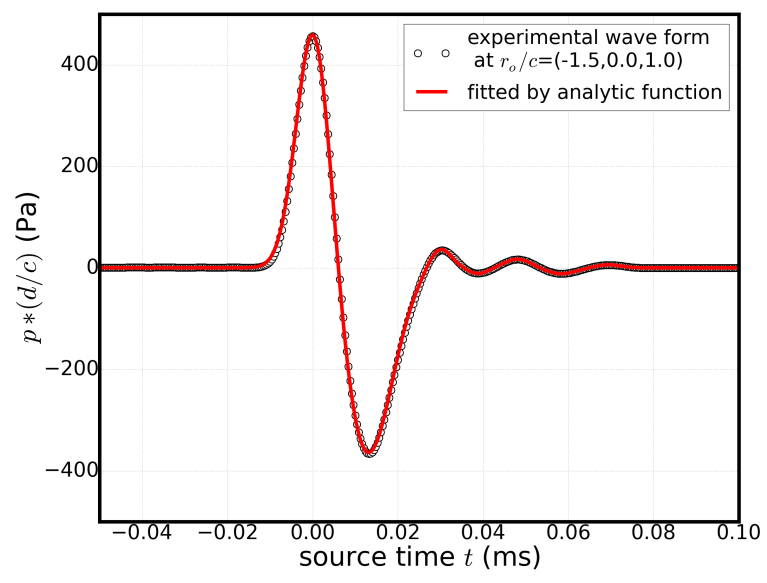

(a)

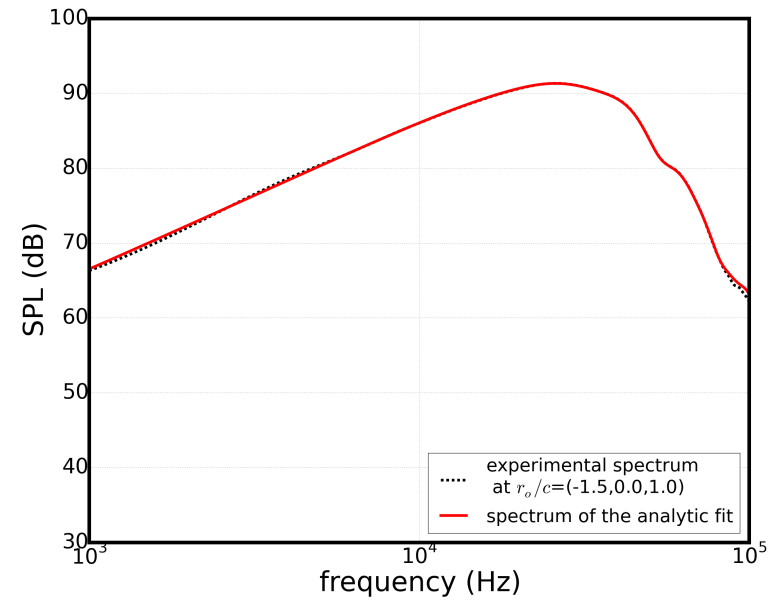

(b)

Figure 6: Comparison of the analytically modeled and measured incident waveforms at observer point $r_{o}=$ $(-1.5 c, 0, c)$. (a) $p \times(d / c)$ vs. time; (b) SPL spectrum of the experimental and modeled source function.

A. Case 75c: source point at $75 \%$ chord, $r_{q}=(-0.25 c, 0,-0.157 c)$

Computational results for the case where the point source is placed at $75 \%$ chord are presented first. For these predictions, the computational mesh for the wing surface is Mesh \#2 (see Table 1) with a total of 590,724 elements. In the time domain boundary element method, surface integration over each element is computed by first mapping the element into a standard two-dimensional element of $[-1,1] \times[-1,1]$ and then evaluating the double integral by the Legendre-Gauss quadrature rule of degree six in each dimension. The time domain simulation is started at the initiation of the point source and lasts sufficiently long such that the scattered waves within the computational domain become negligibly small. For the current computation, the nondimensional time step is $a \Delta t / c=0.002$, where $a$ is the speed of sound and $c$ is the chord length. The temporal basis function is the optimized 6-point 4th-order scheme (50-opt-1-0.6) as detailed by $\mathrm{Hu}, \frac{69}{6}$ which has an expected frequency resolution up to $f_{\text {max }}=1 / 8 \Delta t \approx 106 \mathrm{kHz}$. A complete solution entails up to 5000 time steps and example instantaneous pressure contours at intermediate time steps are shown in Figure 7. Scattering of the acoustic pulse by the leading and trailing edges of the wing are clearly visible.

After the time domain simulation is complete, the frequency domain solution can be obtained from the result by either using the FFT algorithm or the following summation:

$$
p(r, \omega)=\Delta t\left[p\left(r, t_{1}\right) e^{i \omega t_{1}}+p\left(r, t_{2}\right) e^{i \omega t_{2}}+p\left(r, t_{3}\right) e^{i \omega t_{3}}+\cdots \cdots+p\left(r, t_{N_{t}}\right) e^{i \omega t_{N_{t}}}\right]
$$

where $\Delta t$ is the time step for the simulation and $N_{t}$ is the total number of time steps. One of the advantages of the time domain approach is that solutions at all frequencies within the temporal resolution are available after a single time domain simulation. Figure 8 shows two examples of the frequency domain solution. Plotted are the real part of $p(r, \omega)$ at $20 \mathrm{kHz}$ and $40 \mathrm{kHz}$. Patterns of scattered waves, wave interference, and shielded zones are clearly visible.

\section{Comparison in the time and frequency domains}

As mentioned in Section IV, the pressure history along two parallel lines of far field observer points are computed and recorded. The far field observer points are located at coordinates $r_{o}=(x, 0, c)$ and $r_{o}=(x, 0,2 c),(-3 \leq x / c \leq 3)$ and coincide with the lines of microphones in the QFF experiment. In TD-FAST computations, far field observer points can be placed freely and generally do not incur significant additional computational cost.

Figure 9 shows the acoustic pressure time history at a set of selected points along the line of observers located one chord away $(z=c)$ from the midspan airfoil section. The set of plots include observer locations from $x=-2 c$ (top of the figure) to $x=2 c$ (bottom of the figure) with a constant increment of $0.25 c$ in the streamwise coordinate. For reference, the unshielded direct sound from the point source without the model installed is also plotted as dashed lines. The acoustic signal at these observer points consists mainly of the waves scattered by the leading and trailing edges. 


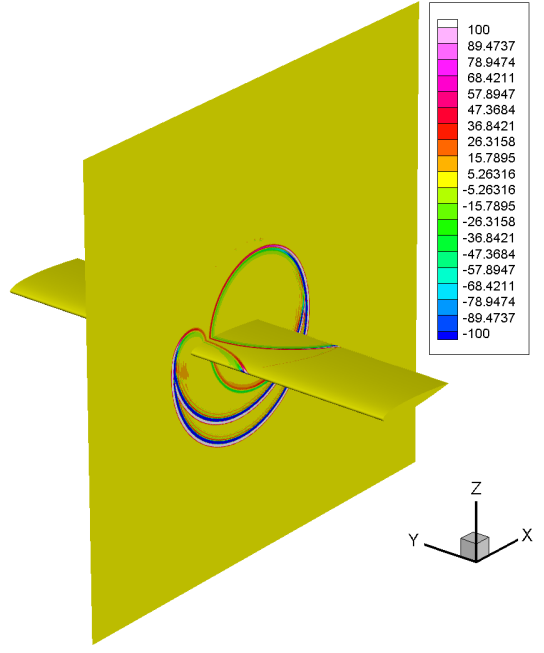

(a)

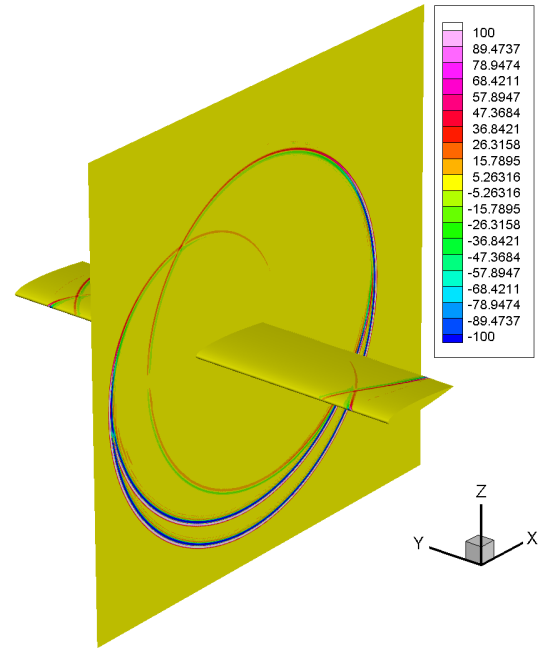

(b)

Figure 7: Pressure contour snapshots at two instances, showing the scattering by the wing leading and trailing edges. The source point is located at $75 \%$ chord at $r_{q}=(-0.25 c, 0,-0.137 c)$. (a) $t=0.54$ (ms); (b) $t=1.1(\mathrm{~ms})$.

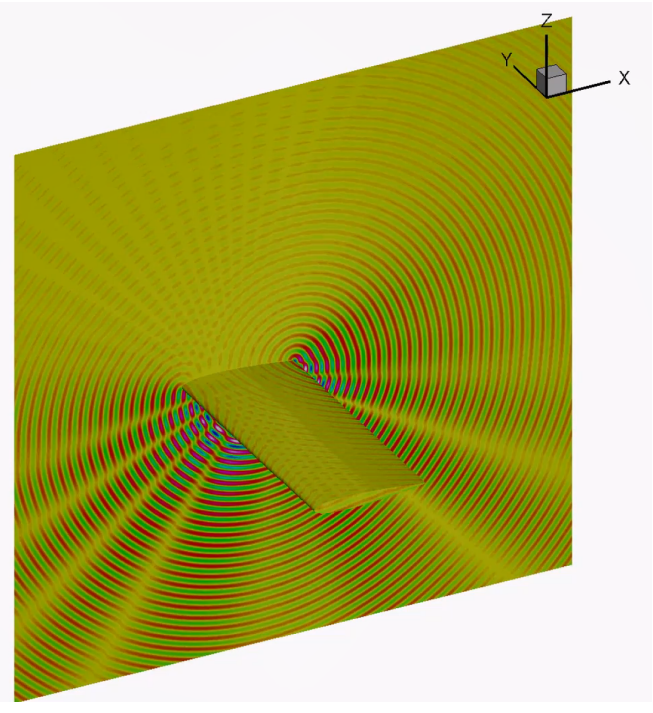

(a)

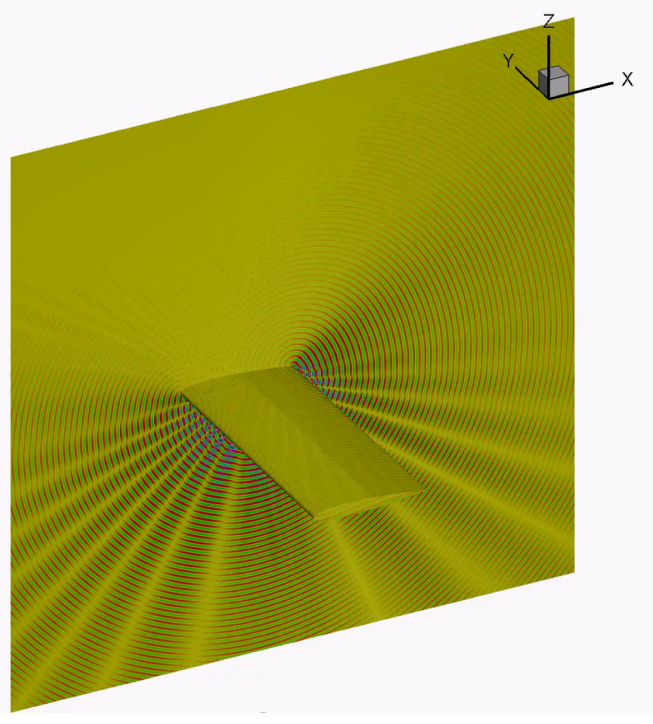

(b)

Figure 8: Example frequency domain solutions converted from the time domain simulation. The source point is located at $75 \%$ chord at $r_{q}=(-0.25 c, 0,-0.137 c)$. (a) $20 \mathrm{kHz}$; (b) $40 \mathrm{kHz}$. 
To aid in analyzing the results, let $t_{A}$ and $t_{B}$ represent the peak arrival times for the waves scattered by the leading and trailing edges, respectively. For an observer point located at $\left(x_{o}, 0, z_{o}\right)$ and a point source located at $\left(x_{q}, 0, z_{q}\right)$, these times may be approximated as

$$
\begin{gathered}
t_{A}=\frac{M\left(x_{q}-x_{o}\right)+\sqrt{\left(c+x_{q}\right)^{2}+\left(1-M^{2}\right) z_{q}^{2}}+\sqrt{\left(c+x_{o}\right)^{2}+\left(1-M^{2}\right) z_{o}^{2}}}{\left(1-M^{2}\right) a} \\
t_{B}=\frac{M\left(x_{q}-x_{o}\right)+\sqrt{x_{q}^{2}+\left(1-M^{2}\right) z_{q}^{2}}+\sqrt{x_{o}^{2}+\left(1-M^{2}\right) z_{o}^{2}}}{\left(1-M^{2}\right) a}
\end{gathered}
$$

(where the source signal peak is assumed to be emitted at $t=0$ ). It can be inferred from Eqs. 11 and 12 (with $M=0$ for the current case) that for points on the observer line $(x, 0, c)$ with coordinate $x$ approximately greater than $-c$, the pressure wave from the trailing edge is observed first and is followed by one from the leading edge. The order is reversed for points with coordinate $x$ approximately less than $-c$. The arrival times for scattered waves from the leading and trailing edges are nearly the same for observer points located around $x=-c$. The effect of shielding can be seen in the amplitude variation of the scattered waves.

It is interesting to note in Figure 9 that some relatively small oscillations are also present around time $t=3(\mathrm{~ms})$, especially for observer points $-1.25 c \leq x \leq-0.25 c$. These waves are due to scattering at the side edges of the wing and are discussed further in Section VIII.

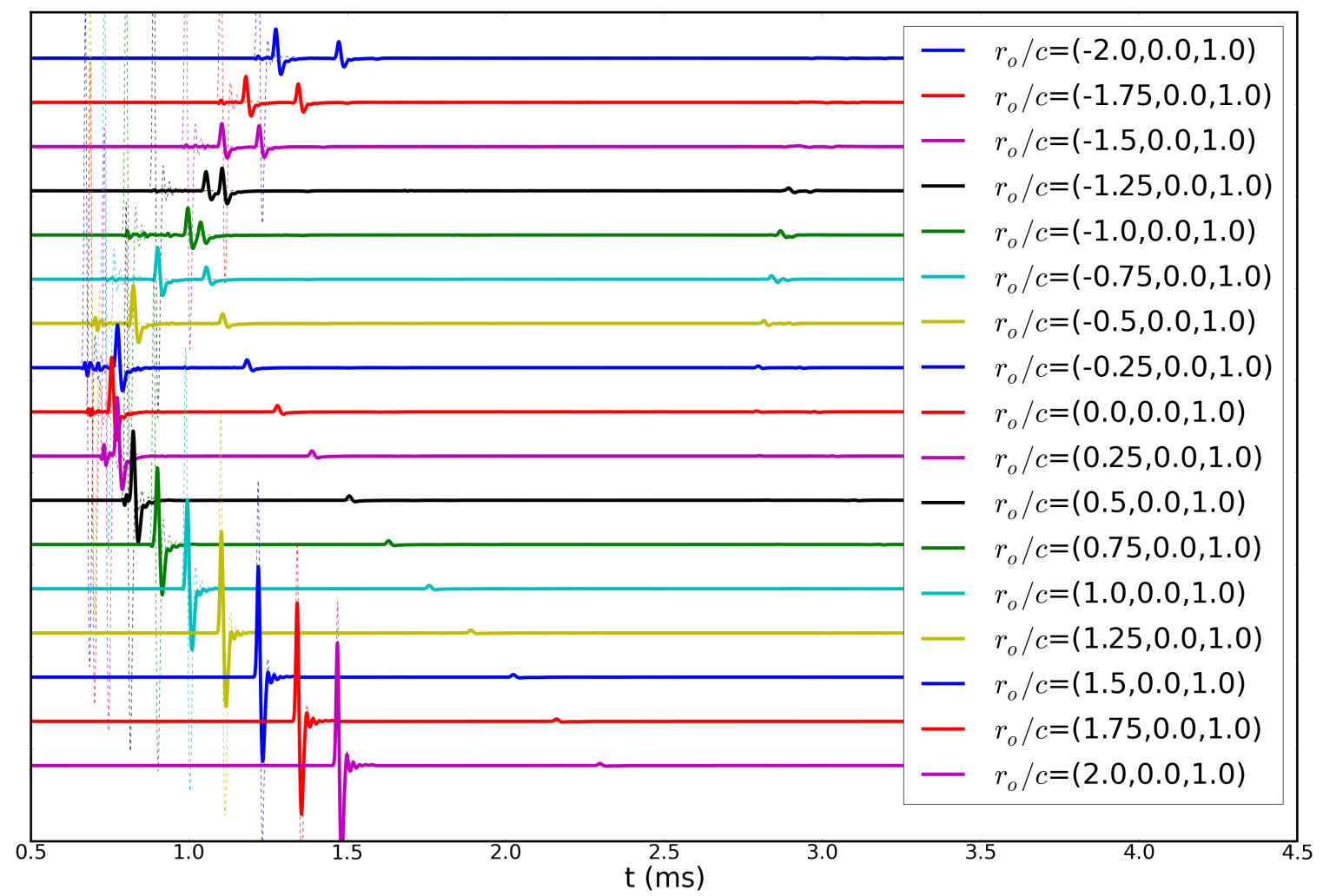

Figure 9: Acoustic pressure time history at selected far field observer points. The graphs are stacked along the streamwise direction from top to bottom of the figure. The source point is located at $75 \%$ chord at $r_{q}=(-0.25 c, 0,-0.137 c)$. Dotted lines indicate unshielded direct sound from the incident source.

Comparisons of the predicted and measured time domain data at four observer locations are provided in Figure 10a. The TD-FAST computational results are shown as purple lines and the QFF measurements are in green. It is seen that computed pressure peak values, as well as the overall shapes of the signal, match the experimental results very well. The small deviations may be partially due to the fact that the experimental results were obtained by averaging over multiple measurements while the computational results represent a single simulation. Figure $10 \mathrm{p}$ provides comparison 
of the predicted and measured sound pressure level (SPL) spectra. Overall, very good agreement is seen over the frequency range from $1 \mathrm{kHz}$ to $100 \mathrm{kHz}$. This demonstrates the advantage of the TD-FAST time domain approach in which solutions for all frequencies within the temporal resolution are computed at once. A notable discrepancy is seen around $40 \mathrm{kHz}$ where the computational results show a reduction in SPL while no obvious dip is seen in the experimental results. It is believed that differences between the source waveform model and actual QFF incident source are the main driver for this discrepancy. More specifically, selection of a specific measured source waveform and associated measurement artifacts lead to uncertainty in the correct source waveform model. A closer examination of this discrepancy and the effect of source waveform is presented in Section IX]

\section{Comparison of shielding factor}

As described in previous reports on the NACA 0012 airfoil scattering problem,,$\sqrt[314]{ }$ a shielding factor may be defined as

$$
\eta(f)=\frac{\left|p_{s}(f)\right|}{\left|p_{i}(f)\right|}
$$

where $p_{s}(f)$ and $p_{i}(f)$ are respectively the shielded and unshielded (or incident) pressure at frequency $f$.

An octave band average of the shielding factor has also been defined as

$$
\bar{\eta}\left(f_{c}\right)=\sqrt{\frac{\sqrt{2}}{f_{c}} \int_{f_{c} / \sqrt{2}}^{\sqrt{2} f_{c}} \eta^{2}(f) d f}
$$

where $f_{c}$ is the center frequency of the octave band $\frac{34}{34}$

Figure 11 provides comparison of the predicted and measured shielding factors for the octave bands for center frequencies $f_{c}=7 \mathrm{kHz}(5-10 \mathrm{kHz}), 14 \mathrm{kHz}(10-20 \mathrm{kHz}), 28 \mathrm{kHz}(20-40 \mathrm{kHz})$ and $56 \mathrm{kHz}(40-80 \mathrm{kHz})$. The computational results are shown in purple lines and the experimental values are represented by solid triangles. Overall, the agreement is very good, especially for the octave bands centered at $7 \mathrm{kHz}, 14 \mathrm{kHz}$, and $28 \mathrm{kHz}$. Moreover, as substantially more observer points are included in the TD-FAST computations, the predictions provide additional detail of the shielding factor variation along the observer line, especially within the shadow zone.

\section{B. Case LE: source point at Leading Edge, $r_{q}=(-c, 0,-0.125 c)$}

Next, computational results for the case where the point source is placed near the leading edge of the airfoil are presented. Again, both time domain and frequency domain comparisons with the QFF experimental results will be presented.

Figure 12 provides examples of instantaneous pressure contours illustrating the onset and pattern of scattering by the leading and trailing edges. The point source is located at coordinates $r_{q}=(-c, 0,-0.125 c)$, directly below the leading edge of the airfoil. Examples of frequency domain pressure contours converted from the time domain solution are shown in Figure 13 for frequencies at $10 \mathrm{kHz}$ and $20 \mathrm{kHz}$. For results shown in this section, the computational mesh (i.e., Mesh \#2) and time step are the same as in the previous case.

\section{Comparison in the time domain and the frequency domain}

Figure 14 shows the acoustic pressure time history at selected points along the line of observer points located one chord away $(z=c)$ from the airfoil. The set of plots includes observer locations along the streamwise direction at coordinates from $x=-2 c$ (top of the figure) to $x=2 c$ (bottom of the figure). For reference, the unshielded direct sound from the point source without the model installed is also plotted as dashed lines. Similar to the previous case, the acoustic signal at these observer points consists mainly of waves scattered by the leading and trailing edges. Here, it is seen that for points on the observer line $(x, 0, c)$ with coordinate $x$ approximately less than $1.25 c$, the pressure wave from the leading edge is observed first and followed by one from the trailing edge. The order is reversed for points with coordinate $x$ approximately greater than $1.25 c$. The arrival times for scattered waves from the leading and trailing edges are nearly the same for observer points located around $x=1.25 \mathrm{c}$. Again, waves caused by scattering at the side edges of the wing appear around time $t=3(\mathrm{~ms})$, most notably for observer points $-0.25 c \leq x \leq 0.25 c$.

Comparison of the predicted and measured time domain data at four observer locations are provided in Figure $15 \mathrm{a}$. In general, the peak values and shapes of the predicted pressure time signal (purple lines) match the experimental 

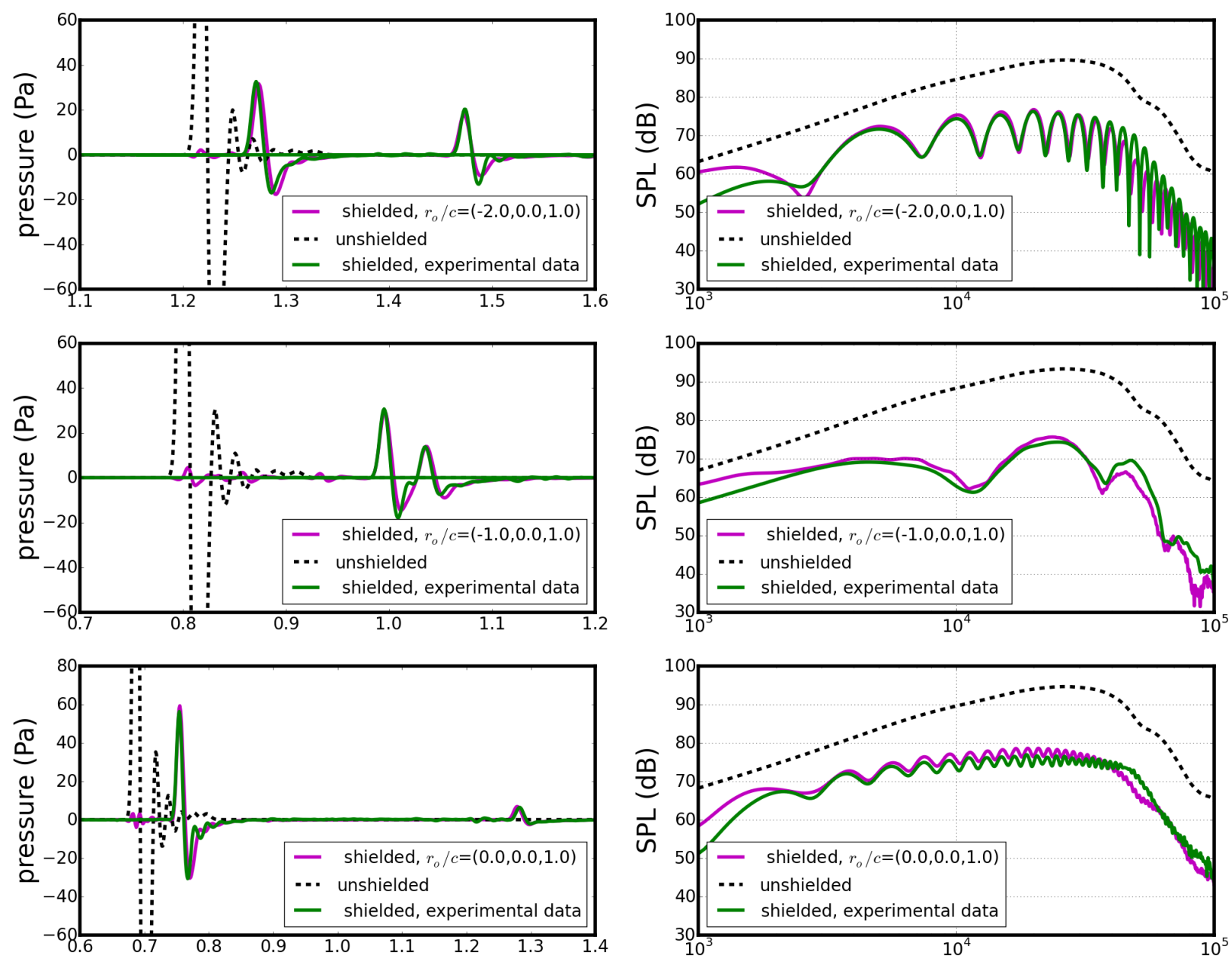

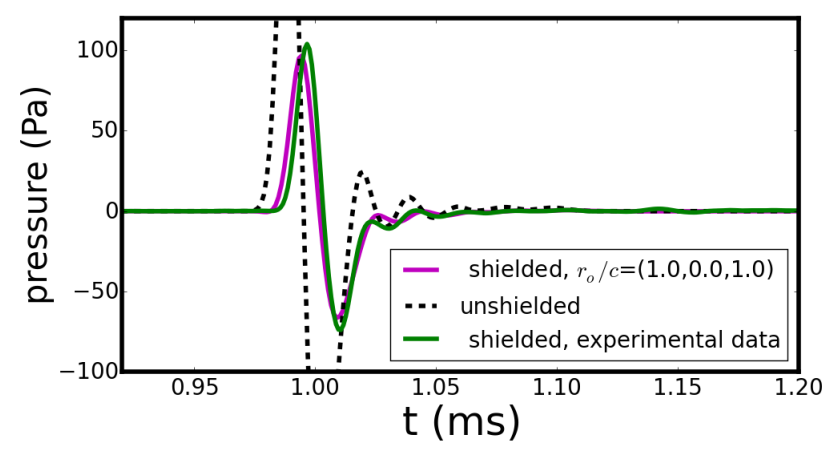

(a)

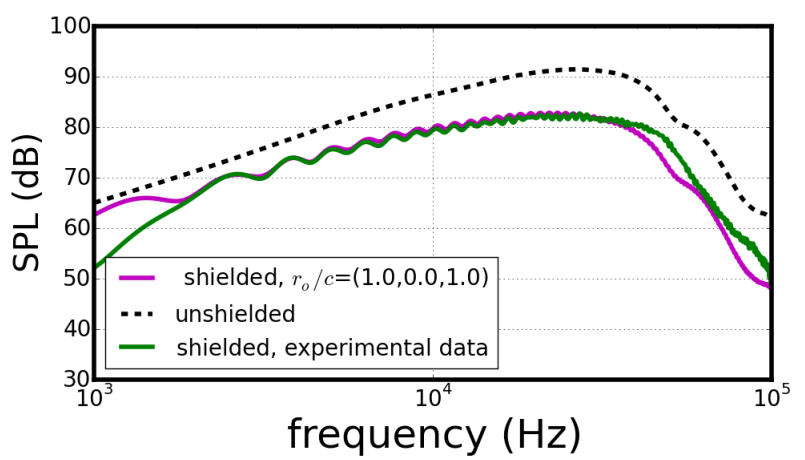

(b)

Figure 10: Comparisons between the computed and measure acoustic pressure at four far field locations as indicated. The source point is located at $75 \%$ chord at $r_{q}=(-0.25 c, 0,-0.157 c)$. Purple lines are the computations and green lines are the measurements. Dotted lines indicate unshielded direct sound from the incident source. (a) time domain; (b) SPL spectrum. 

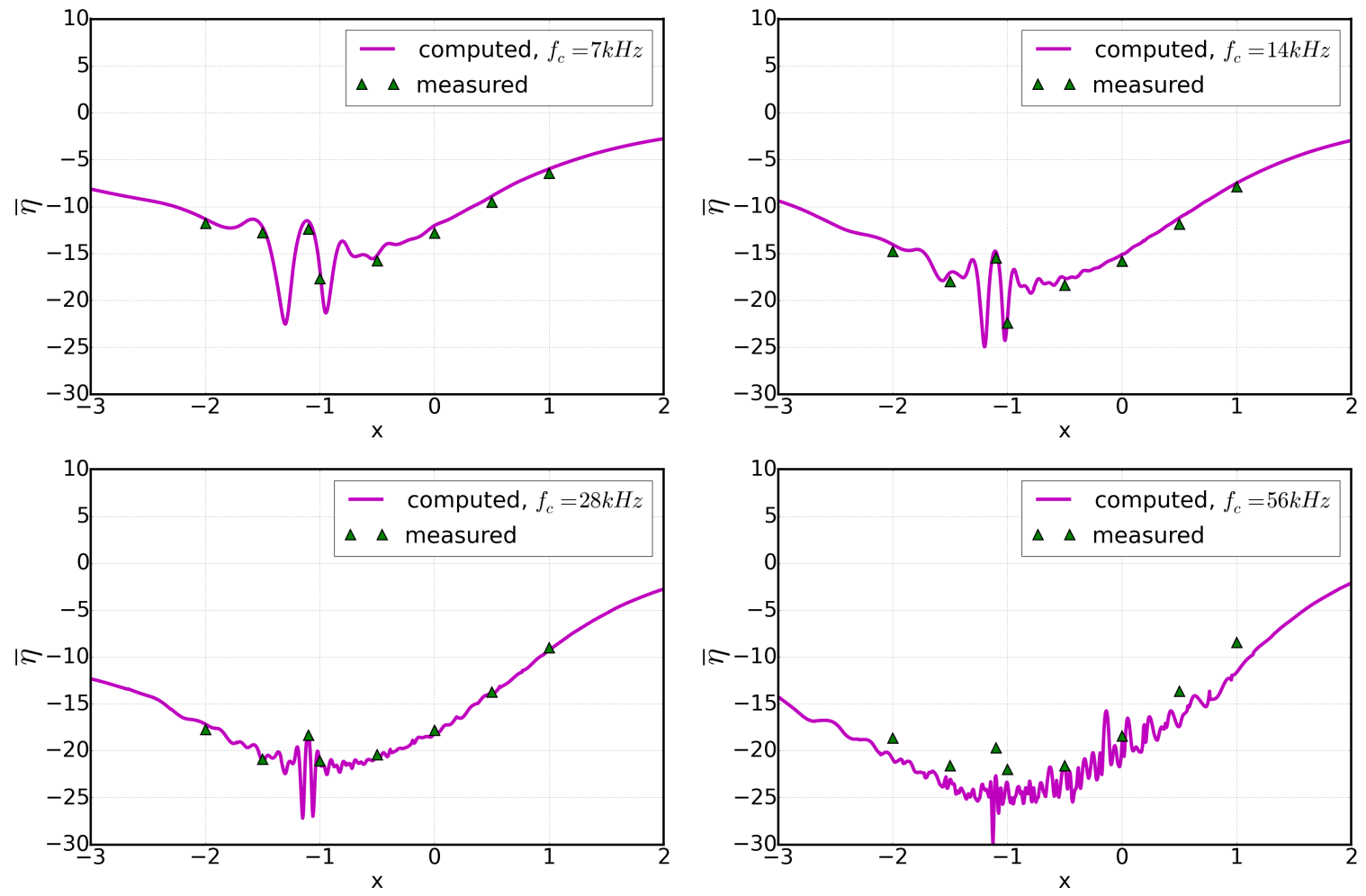

Figure 11: Averaged shielding factor in octave bands as indicated. The source point is located at $75 \%$ chord at $r_{q}=(-0.25 c, 0,-0.157 c)$. The observer line is at $z=c$.

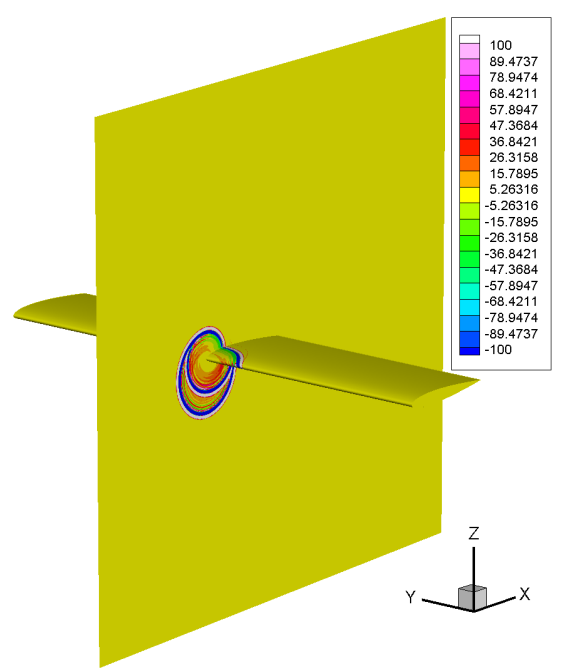

(a)

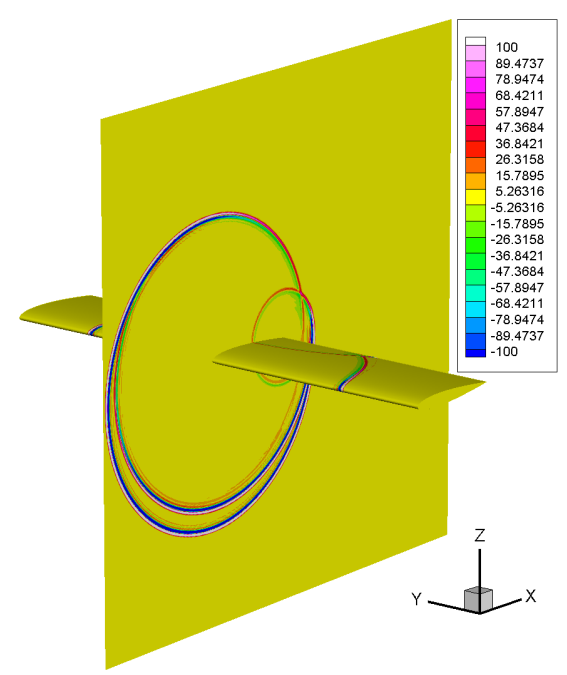

(b)

Figure 12: Pressure contour snapshots at two instances, showing the scattering by the airfoil leading and trailing edges. The source point is located near the leading edge at $r_{q}=(-c, 0,-0.125 c)$. (a) $t=0.23(\mathrm{~ms})$; (b) $t=0.78(\mathrm{~ms})$. 


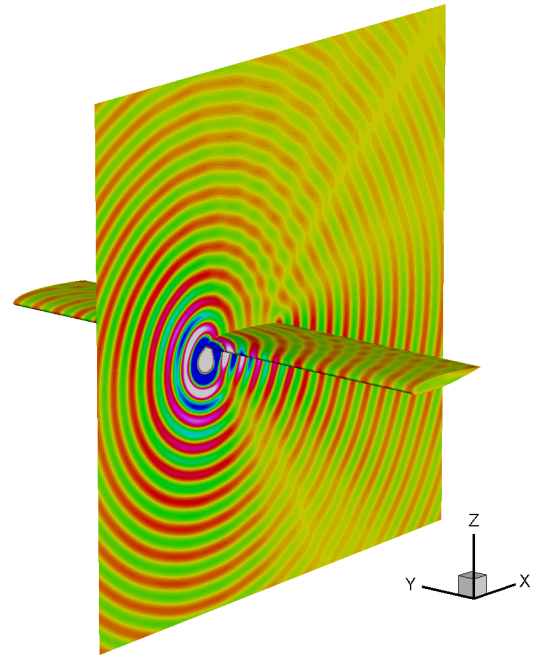

(a)

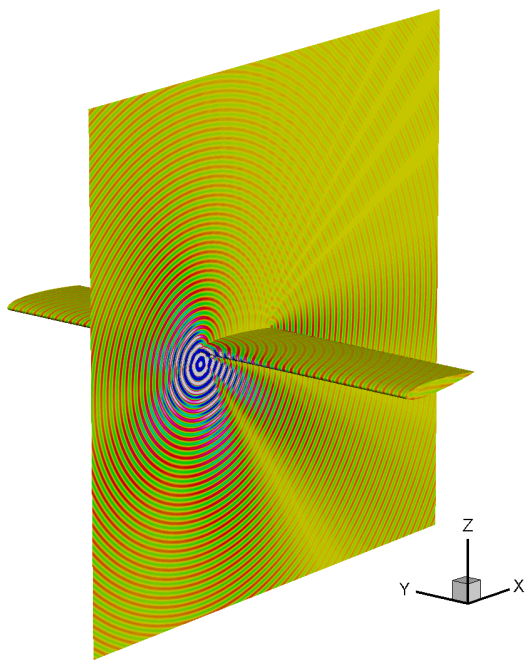

(b)

Figure 13: Example frequency domain solutions converted from the time domain solution. The source point is located near the leading edge at $r_{q}=(-c, 0,-0.125 c)$. (a) $10 \mathrm{kHz}$; (b) $20 \mathrm{kHz}$.

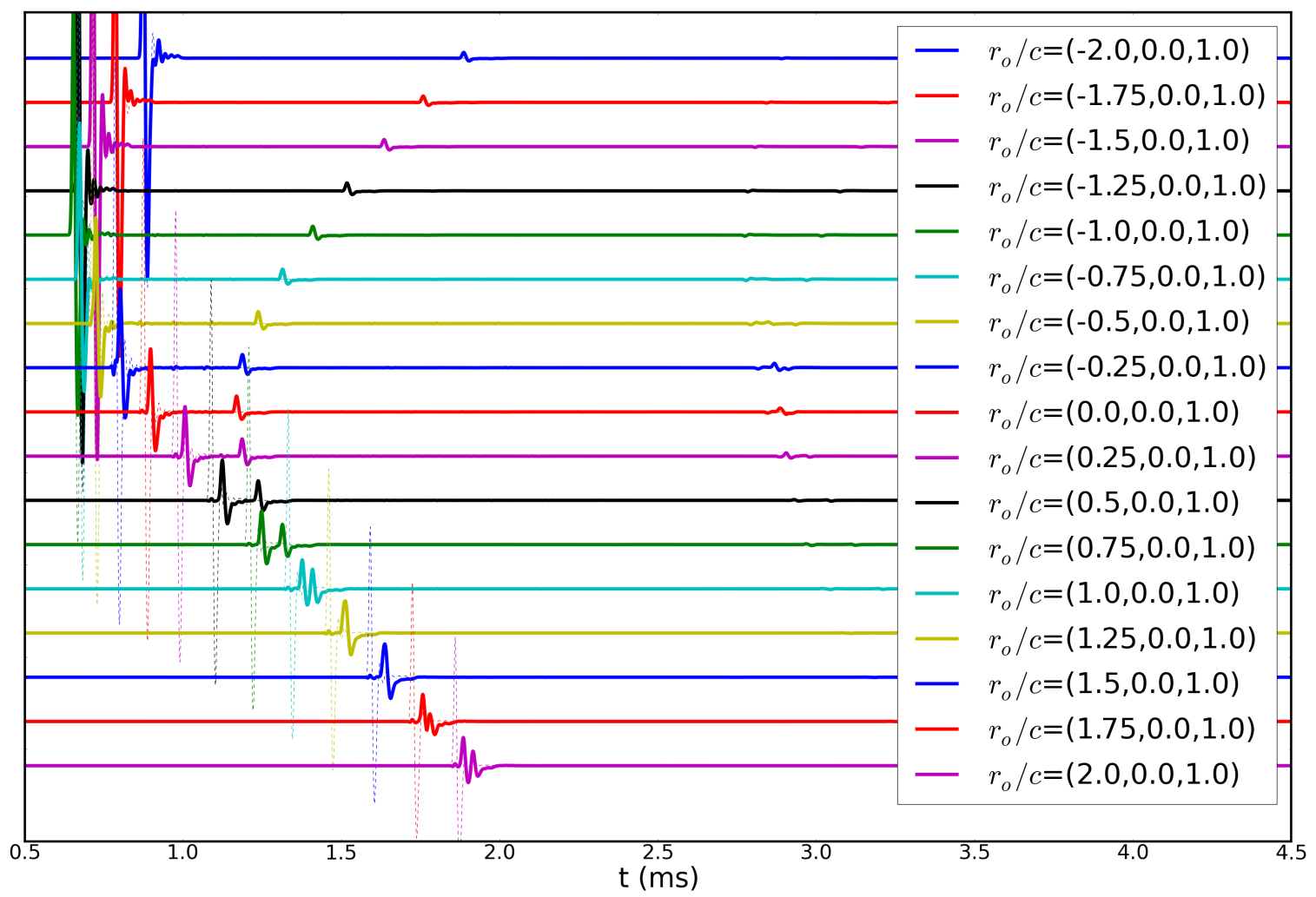

Figure 14: Acoustic pressure time history at selected far field observer points. The graphs are stacked along the streamwise direction from top to bottom of the figure. The source point is located near the leading edge at $r_{q}=$ $(-c, 0,-0.125 c)$. Dotted lines indicate unshielded direct sound from the point source. 
measurements (green lines) fairly well for upstream observers. However, a shift in the peak arrival time is observed for coordinate locations $x=-c, 0$, and $c$.

Figure $15 \mathrm{p}$ provides comparison of the predicted and measured SPL spectra. While the spectra for the observer points upstream of the airfoil $(x=-2 c$ and $x=-c)$ match reasonably well, the results for the two downstream points $(x=0$ and $x=c)$ show substantial differences. After further investigation, it was discovered that the modeled source was located incorrectly for comparison with the QFF experimental setup. In order to maintain a distance of $25 \mathrm{~mm}$ along the shortest distance/normal to the midspan airfoil section at the LE location (as prescribed in the QFF setup), the source coordinate should be $r_{q}=(-c, 0,-0.1507 c)$. It is believed that this difference in computational and experimental source location is the primary cause for the discrepancies in these cases. Additional simulations will be performed using the updated source location. The current results are presented to illustrate the effects of small source location differences, as well as to highlight the subtleties for placement of the LE source location.

\section{Comparison of shielding factor}

For completeness, Figure 16 provides a comparison of the predicted and measured octave band averaged shielding factor $\bar{\eta}\left(f_{c}\right)$ (see Eq. 14) for center frequencies $f_{c}=7 \mathrm{kHz}, 14 \mathrm{kHz}, 28 \mathrm{kHz}$ and $56 \mathrm{kHz}$. The computational results are shown in purple lines and the experimental values are presented as solid triangles. Overall, the predicted and measured quantities compare quite well and it appears that the integrated shielding factor is less affected by the slight difference in source location.

C. Case 70c: source point at 70\% chord, $r_{q}=(-0.3 c, 0,-0.237 c)$

In the third case, computational results for the point source located at $70 \%$ chord at $r_{q}=(-0.3 c, 0,-0.237 c)$ are presented. This is a benchmark case that has been studied experimentally and documented by multiple research teams $\frac{3,4}{3}$ As a benchmark problem, results from different facilities were standardized and compared in terms of the octave band averaged shielding factor $\bar{\eta}\left(f_{c}\right)$ as defined in Eq. 14 .

Figure 17 provides comparison of $\bar{\eta}\left(f_{c}\right)$ for the octave band center frequencies $f_{c}=7 \mathrm{kHz}, 14 \mathrm{kHz}, 28 \mathrm{kHz}$ and $56 \mathrm{kHz}$ without mean flow. The observer points are located along a far field line that is two chords away from the airfoil, namely with coordinates $r_{o}=(x, 0,2 c)$. In this figure, TD-FAST predictions are plotted as purple lines and experimental measurements ${ }^{4}$ are represented as black symbols/lines. The predicted and measured shielding factors are in very good agreement. Again, because substantially more observer points are included in the simulation, the TD-FAST results provide further details on the shielding factor variation along the observer line, especially in the shadow zone.

Figure 18 shows the comparison of $\bar{\eta}\left(f_{c}\right)$ for the octave bands center frequencies $f_{c}=7 \mathrm{kHz}, 14 \mathrm{kHz}, 28 \mathrm{kHz}$, and $56 \mathrm{kHz}$ in the presence of a mean flow with Mach number, $M=0.16$. The level and trend for the shielding factor along the observer line $(z=2 c)$ are in very good agreement for the octave bands centered at $f_{c}=14 \mathrm{kHz}$ and $28 \mathrm{kHz}$. On the other hand, substantial differences are observed in the results for the first and last octave bands, i.e., $f_{c}=7 \mathrm{kHz}$ and $56 \mathrm{kHz}$. For the first octave band centered at $f_{c}=7 \mathrm{kHz}$, the experimental data may be distorted by low-frequency contamination in the wind tunnel when the mean flow is present as discussed by Rossignol et al ${ }^{[4}$ Interestingly, the current TD-FAST computation matches the DLR numerical simulation well. For the last octave band centered at $f_{c}=56 \mathrm{kHz}$, the current TD-FAST computation shows a higher degree of shielding compared to the experimental values and the DLR simulation. A possible explanation might be that the mean flow is assumed to be constant in the TD-FAST computation while the effects of boundary layer are included in both the experiments and DLR simulation. As noted by Rossignol et al., ${ }^{4}$ refraction through the boundary layer may contribute to the increase of the acoustic wave in the shadow zone at high frequencies.

\section{Computational mesh refinement effects}

To study the effect of mesh size on the computational results, three surface meshes were constructed (referred to as Mesh \#1, \#2, and \#3) as detailed in Section IV Figure 19 shows an example of solutions computed using the three meshes for the $75 \mathrm{c}$ case. The time step for all the three computations is the same $(a \Delta t / c=0.002)$, so that the temporal resolution up to $100 \mathrm{kHz}$ is maintained. The total number of elements are 183,$700 ; 590,724$; and 965,656 , respectively for the three meshes. On a cluster of 16 GPUs (Nvidia V100 model), computational time for the three meshes is 

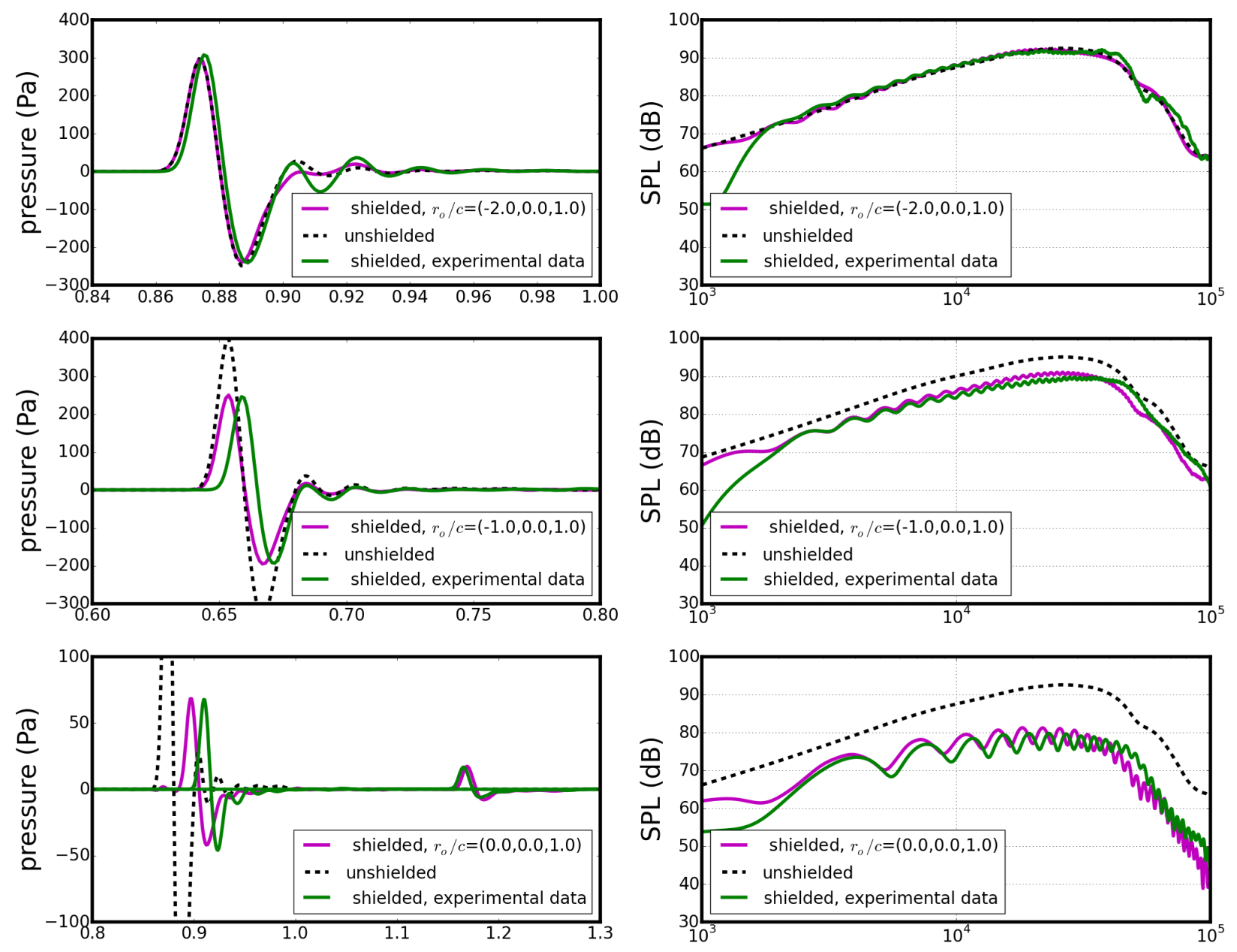

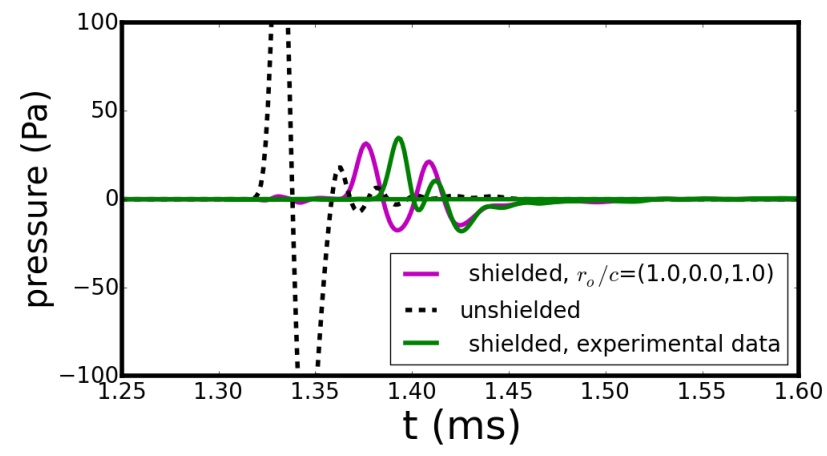

(a)

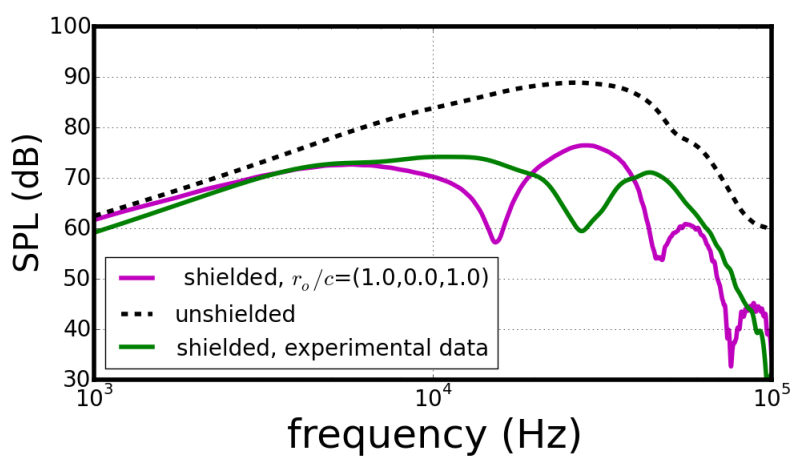

(b)

Figure 15: Comparisons between the computed and measure acoustic pressure at four far field locations as indicated. The source point is located near the leading edge with coordinates $r_{q}=(-c, 0,-0.125 c)$. Purple lines are the computations and green lines are the measurements. Dotted lines indicate unshielded direct sound from the point source. (a) time domain; (b) SPL spectrum. Note: subsequent analysis revealed that the computation source was incorrectly located for the case and comparisons are included for illustrative purposes. 

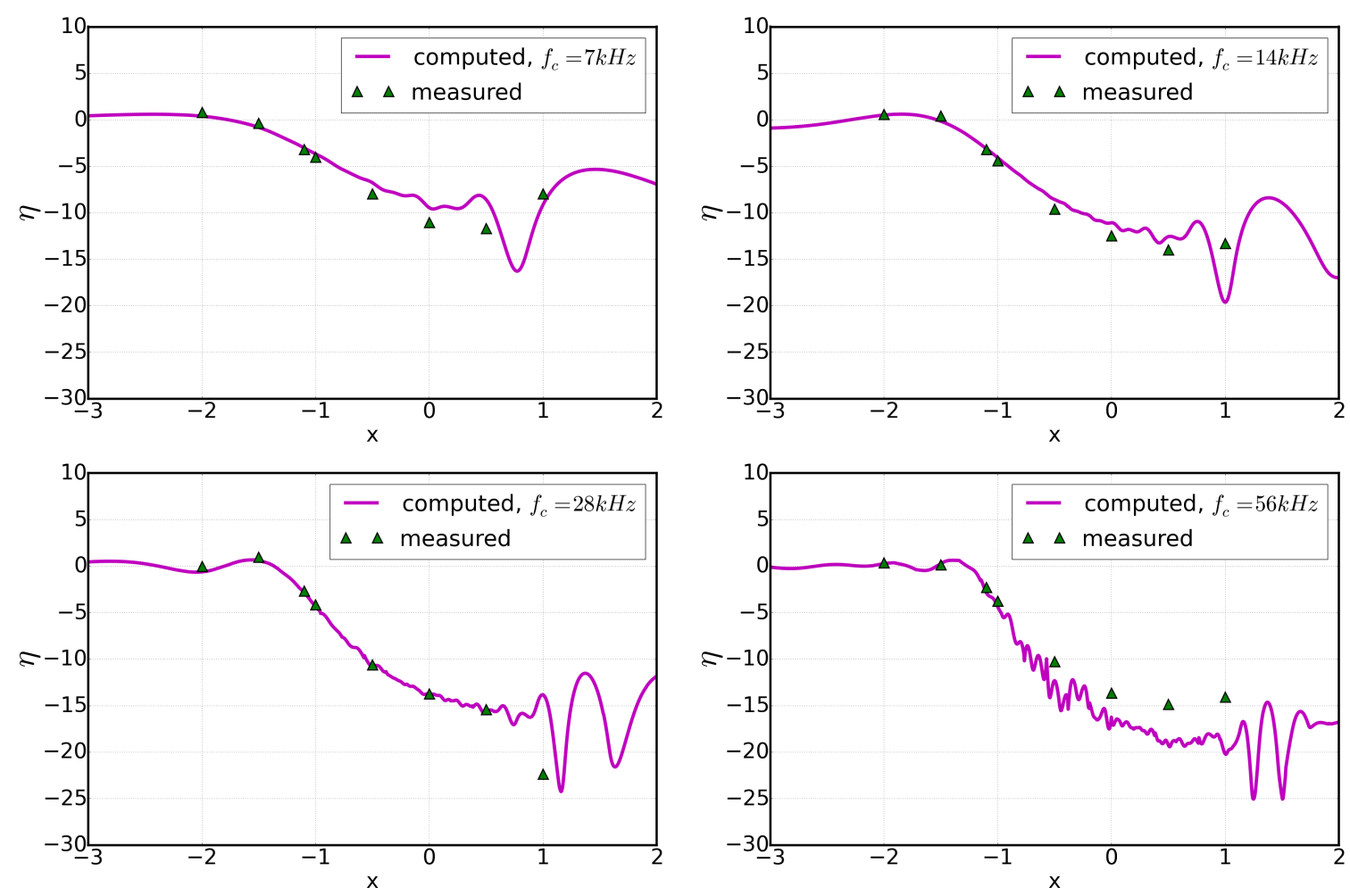

Figure 16: Averaged shielding factor in octave bands as indicated. The source point is located near the leading edge with coordinates $r_{q}=(-c, 0,-0.125 c)$. The observer line is at $z=c$. Note: subsequent analysis revealed that the computation source was incorrectly located for the case and comparisons are included for illustrative purposes. 

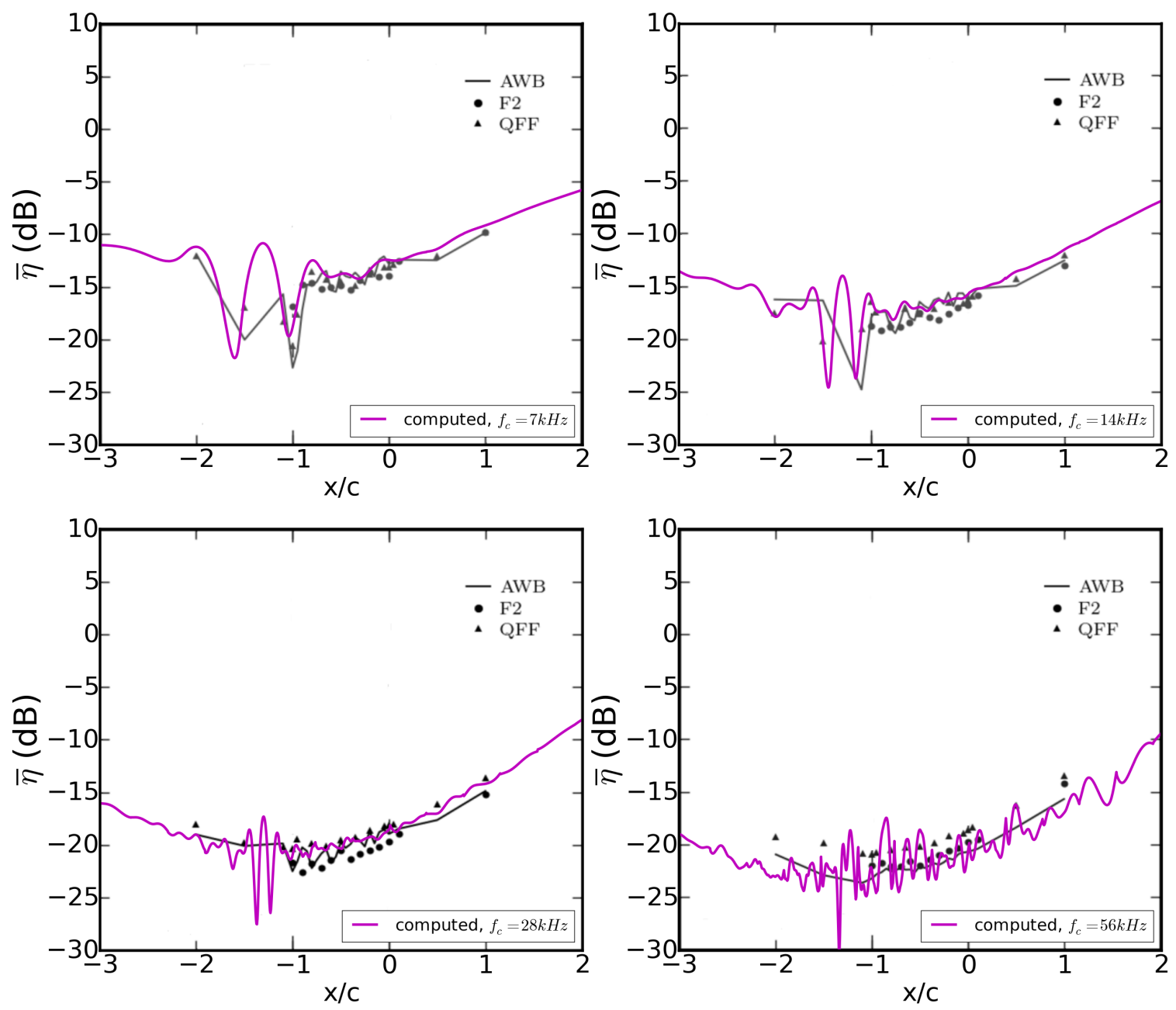

Figure 17: Comparison of computed and measured ${ }^{4}$ octave band averaged shielding factor with no mean flow $(M=0)$. Computational results are plotted in purple and experimental results in black in the identical style as Figure 11 found

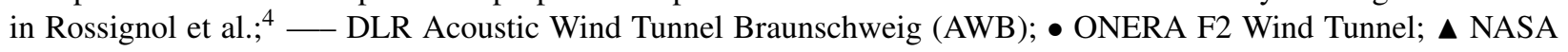
Quiet Flow Facility (QFF). 

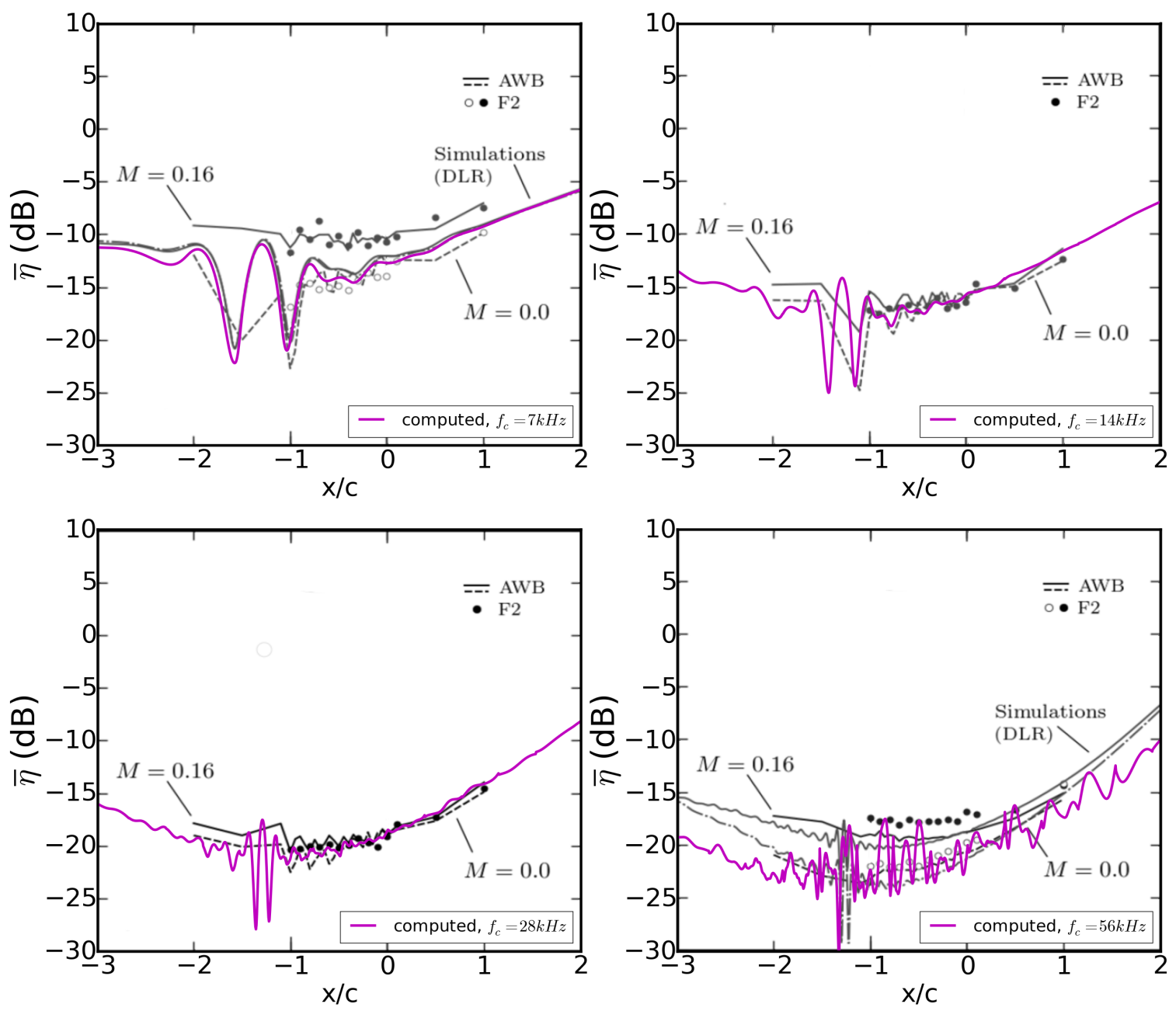

Figure 18: Comparison of computed and measured ${ }^{4}$ octave band averaged shielding factor with mean flow $(M=0.16)$. Computational results are plotted in purple and experimental results in black in the identical style as Figure 12 found in Rossignol et al., 4 — DLR Acoustic Wind Tunnel Braunschweig (AWB); • ONERA F2 Wind Tunnel. Also included are the numerical simulation results from DLR's CAA code PIANO ${ }^{4}$ as indicated. 
respectively 24, 108, and 214 seconds per time step. This approximately follows the scaling of $O\left(N^{1.25}\right)$ per time step where $N$ is the total number of elements. 1$]$

Interestingly, the computational results using the three meshes are seen to be remarkably similar despite the large differences in the total number of elements used for the solution of the boundary integral equation. This may partly be due to the fact that the time step is kept the same for all three meshes and surface integration on each element is done by high-order quadrature. On the left of Figure 19, pressure time histories are shown at two observer points, with coordinates $(-2 c, 0, c)$ and $(-c, 0, c)$, respectively. Labels "A" and "B" denote the peak arrival times of waves scattered by the leading and trailing edges, respectively. The solutions using the three meshes largely coincide, except for the part of the wave labeled " $\mathrm{C}$ " in the figure. This is the direct sound from the point source, which should have been completely blocked by the wing had the numerical solution of the integral equation been error free. Apparently, the most noticeable effect of the mesh size is that this error is reduced as the surface mesh is refined from Mesh \#1 to Mesh \#3. This erroneous wave can also be easily excluded in the post-processing stage by using a windowing that includes only the wave scattered by the leading and trailing edges, in accordance with the estimated signal arrival times given in Eqs. 11 12 The "windowed" frequency domain solutions are shown on the right of Figure 19 for the two observer points. Results for all meshes are generally in very good agreement. Further detail on the differences at higher frequencies are shown in the refined plot of Figure 20 It is seen that the results for Mesh \#2 and \#3 match closely for all the frequencies up to $100 \mathrm{kHz}$. The solution for Mesh \#1 appears to be acceptable even up to perhaps $80 \mathrm{kHz}$.
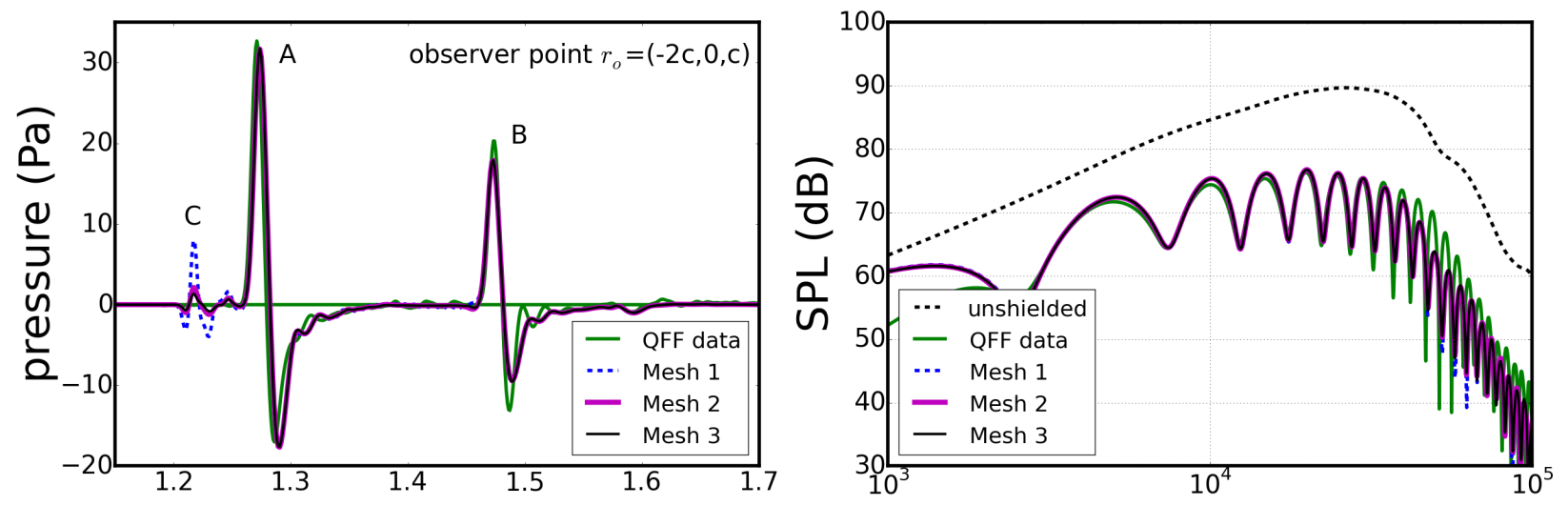

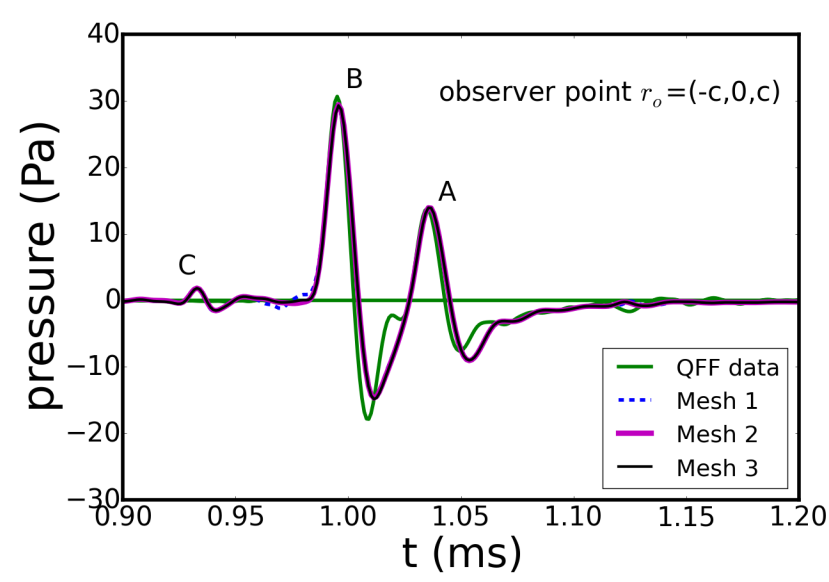

(a)

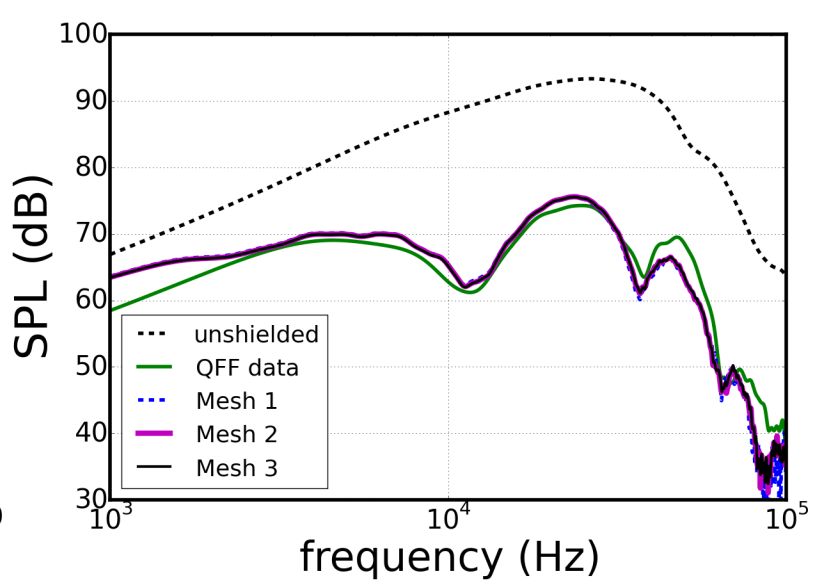

(b)

Figure 19: A comparison of numerical solutions using the three different meshes as indicated. (a) Time domain solution, where points A and B denote the waves scattered by the leading and trailing edges, respectively, and point $\mathrm{C}$ indicates the erroneous direct sound from the point source; (b) Corresponding SPL spectrum (part C is excluded in the computation of the spectrum). 

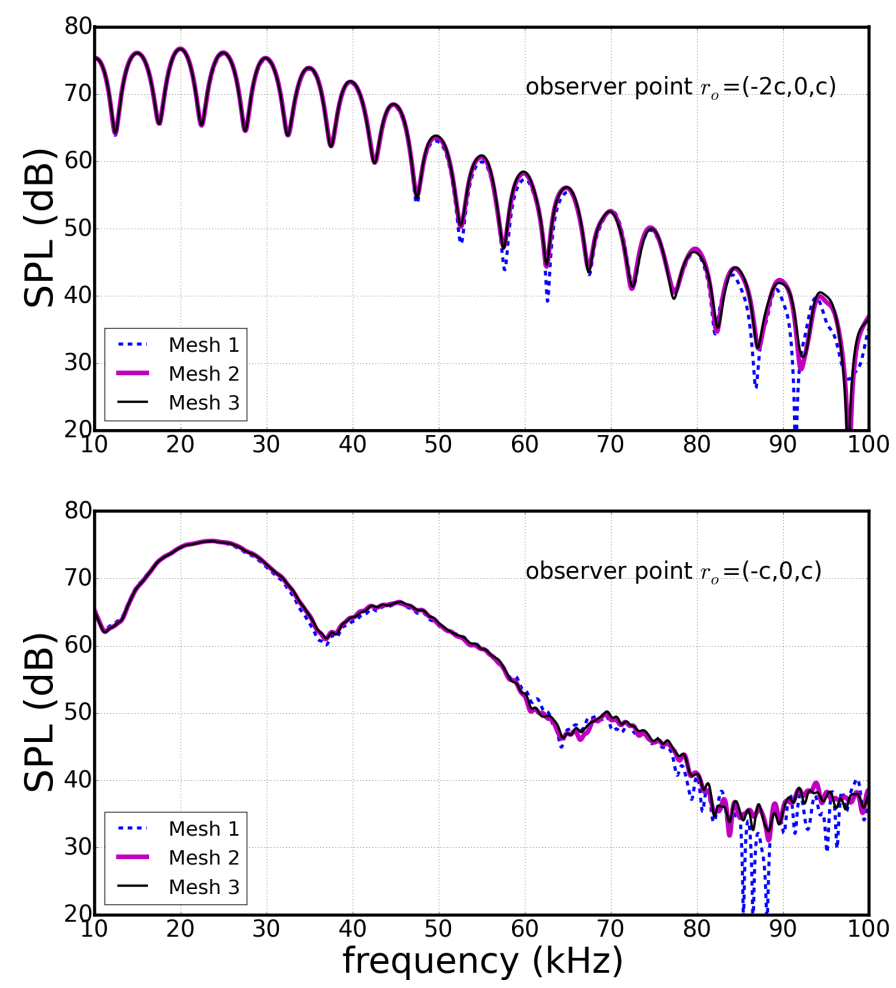

Figure 20: An enlargement of Figure 19 (b), showing a detailed comparison of the SPL spectrum computed using the three different meshes.

\section{Side edge scattering}

As mentioned in Section $\mathrm{VI}$, the finite length of the wing in the spanwise direction leads to wave scattering by the wing side edges. In the computation, the span of the wing is taken to be the same as the QFF model, namely, $4.55 c$, where $c$ is the chord. Figure 21 (top) shows an example of the computed pressure history where the side-edge scattered wave is included. Compared to waves scattered by the leading and trailing edges, the waves scattered by the side edges have a delayed arrival time and smaller amplitude. As seen in Figure 9 , the side-edge scattered waves are more pronounced for observer points above (or nearly above) the wing. For the results shown in Figure 21, the source point is located at $75 \%$ chord (Case $75 \mathrm{c}$ ) and the observer point is located at $r_{o}=(-c, 0, c)$, i.e., one chord length above the leading edge. Since this observer point is located directly above the midspan airfoil section $(y=0)$, scattered waves from both side edges of the wing arrive at the same time. In the QFF experiments, because the laser-induced source pulse is emitted repeatedly and at a high frequency, scattering by the side edges (and side walls) is not included in the spectral analysis of the far field microphone measurements. For the purpose of comparing the computational results with that of the experiments, the side-edge scattered waves are also not included in the frequency domain predictions in all the previous sections. Figure 21.bottom) shows an example SPL spectrum when the side edge scattering is included. The main contribution of side-edge scattered waves appears to be the addition of an oscillation in the spectrum on top of those produced by the leading and trailing edges. Depending on the frequency, the effect of side-edge scattered waves can be as much as $10 \mathrm{~dB}$ for the case shown in Figure 21. The example in this section also shows another advantage for the time domain simulation: the waves scattered by the leading and trailing edges as well as the side edges can be separately identified.

\section{Effect of source waveform}

As noted previously, the scattered SPL spectrum computed by TD-FAST shows a reduction, or a dip, around 40kHz whereas no such reduction is seen in the measurements (for instance, see Figs. 10 and 15). A close inspection indicates 

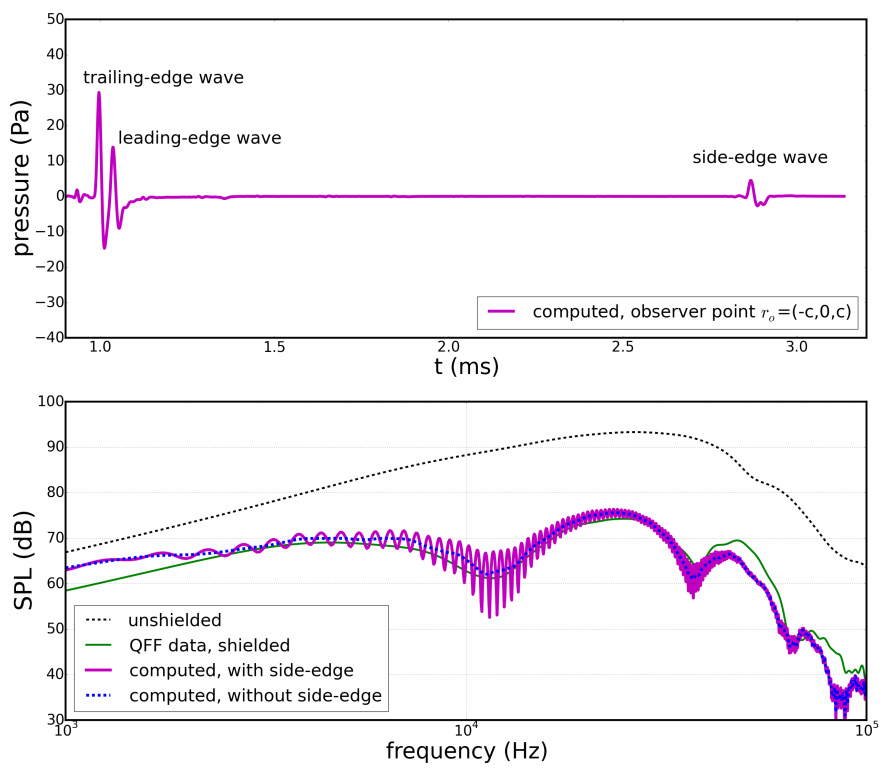

Figure 21: Time domain and frequency domain solutions showing the effect of waves scattered by the side edges of the wing. Top: time history of acoustics pressure at far field point $r_{o}=(-c, 0, c)$; Bottom: comparison of SPL spectrum with and without side edge waves.

that the dip is apparently related to the fact that there is a corresponding reduction in the measured source waveform around $40 \mathrm{kHz}$. As such, a corresponding reduction in SPL of the predicted scattered waves around this frequency may not be unexpected. However, if this characteristic of the measured source waveform is a measurement artifact (e.g., microphone grid cap or bullet nose, angle of incidence), then it would not be present in the true QFF incident waveform and would lead to discrepancies when comparing predicted and measured scattered fields. To confirm that the behavior is indeed a direct result of the source waveform assumed in the computation, an additional simulation was conducted. In this case, a new source waveform was employed in which the dip in level around $40 \mathrm{kHz}$ was removed. The new waveform consists of only the first peak and trough of the measured incident pressure signal, as shown in Figure 22a. Specifically, the new source time function $q_{2}(t)$ retains only the first two terms of $q_{Q F F}(t)$ given in Eq. 9 and therefore, has the following form:

$$
q_{2}(t)=c\left[491.71 e^{-30426(t-0.00035)^{2}}-367.71 e^{-14862(t-0.01291)^{2}} .\right]
$$

The frequency spectrum of the new source function is shown in Figure $22 \mathrm{~b}$ and it does not exhibit an SPL reduction around $40 \mathrm{kHz}$.

Figure 23 shows the effects of using the new waveform (Eq. 15) for the $75 \mathrm{c}$ case. In particular, comparisons of the TD-FAST predicted and QFF measured time and frequency domain results are plotted in the same manner as in Figure 10 No obvious dip in SPL around $40 \mathrm{kHz}$ is observed for the computational results (in purple), consistent with the new source waveform (shown in dashed lines).

A comparison of the two source waveforms (Eqs. 9 and 15 , suggests that the dip in the $q_{Q F F}(t)$ spectrum is likely due to the presence (and/or character) of the secondary oscillations that follow the first peak and trough of the main pulse. So, in addition to potential measurement artifacts, it is possible that the small secondary waves are also slightly affected by atmospheric variations. Regardless of the precise cause, as seen in Figure 5b, the secondary waves are not as uniform as the main pulse and are more location dependent. As such, the waveform measured at the far field microphone locations may not be the true incident wave that impinges on the surface of the airfoil, particularly in regard to the secondary oscillations of the waveform. As the secondary waves contribute most notably to frequencies over $40 \mathrm{kHz}$, discrepancies between the computed and measured SPL spectrum for frequencies higher than $40 \mathrm{kHz}$ are perhaps not unexpected.

It is interesting to note that the shielding factor is not greatly effected by the inclusion of the secondary waves in the source waveform (which remains omnidirectional), as long as the waveform has a sufficiently wide spectrum 


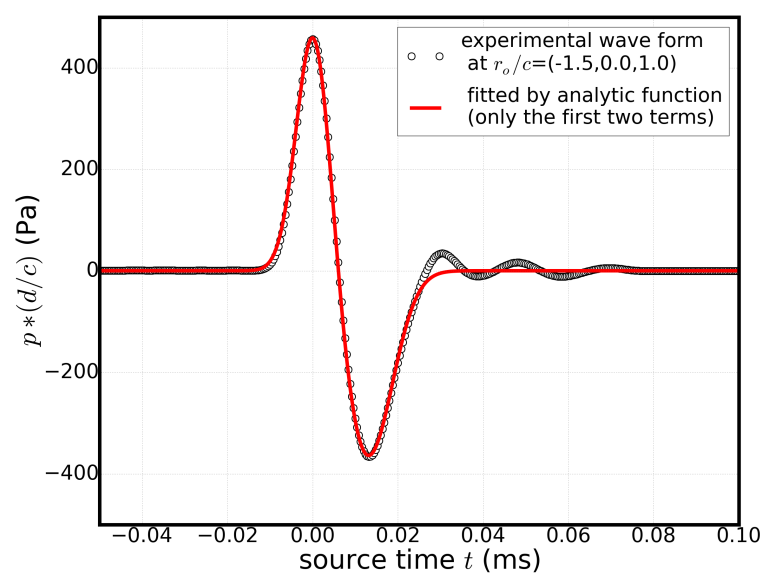

(a)

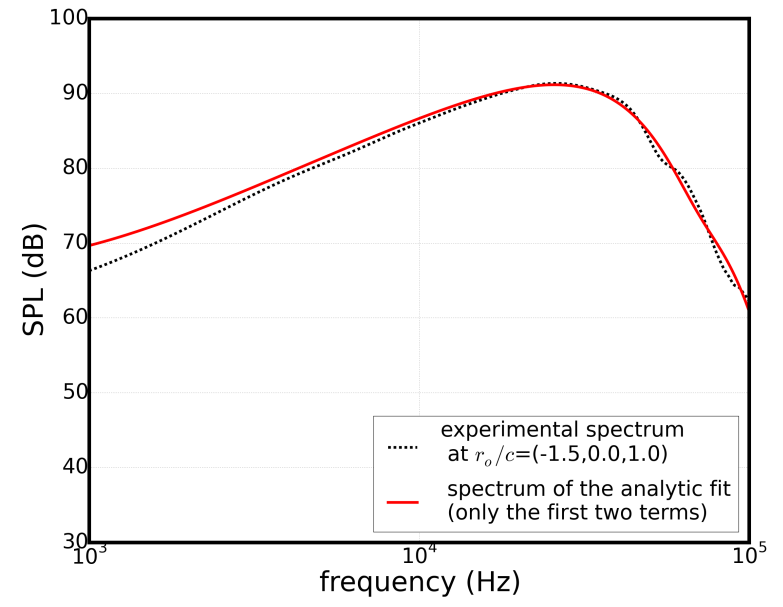

(b)

Figure 22: The source waveform using only the the first two terms of Eq. 9 and fitting the first peak and trough of the measured incident wave at observer point $r_{o}=(-1.5 c, 0, c)$. (a) $p \times(d / c)$ vs. time; (b) SPL spectrum of the experimental and new source function.

that covers the entire frequency range of interest and the time step is small enough to resolve the highest frequency of interest.

\section{Concluding Remarks}

This paper describes the further validation of a time domain boundary element approach for the prediction of the interactions between incident noise sources and nearby aircraft structures. Predictions were completed for multiple source locations and comparisons of these results with measured data presented. Overall, very good agreement between the predicted and measured quantities was obtained in both the pressure time histories and pressure spectra. Some discrepancies were documented at higher frequencies, but were attributed to the selection of the source waveform model.

Comparisons of an integrated shielding factor were also performed and excellent agreement was obtained for cases that did not include a mean flow. This was also the case in the midfrequency range for the case in which a mean flow was included. However, there were some shielding factor discrepancies observed for the lowest and highest octave band center frequencies considered. For the lowest frequency case, distortion of the experimental data by low frequency wind tunnel contamination in the presence of mean flow was identified as a suspected cause. The discrepancies at the highest octave band center frequency remain under investigation.

The effects of surface mesh resolution were also investigated by using three meshes designed to resolve frequencies up to $40 \mathrm{kHz}, 80 \mathrm{kHz}$, and $100 \mathrm{kHz}$, respectively. Interestingly, the computational results using the three meshes were seen to be remarkably similar despite the large differences in the total number of elements. However, this may partly be due to the fact that the time step and high-order surface integration was kept the same for all three meshes.Generally, the coarse mesh performed better than expected at the higher frequencies and the results for the two finer meshes matched closely for all the frequencies up to $100 \mathrm{kHz}$.

Finally, the investigation of additional scattering mechanisms afforded by the time domain approach and source waveform effects were also presented. The very promising results demonstrate the capabilities of the time domain methodology employed in this study and provide further confidence in its continued development and application in future studies. 

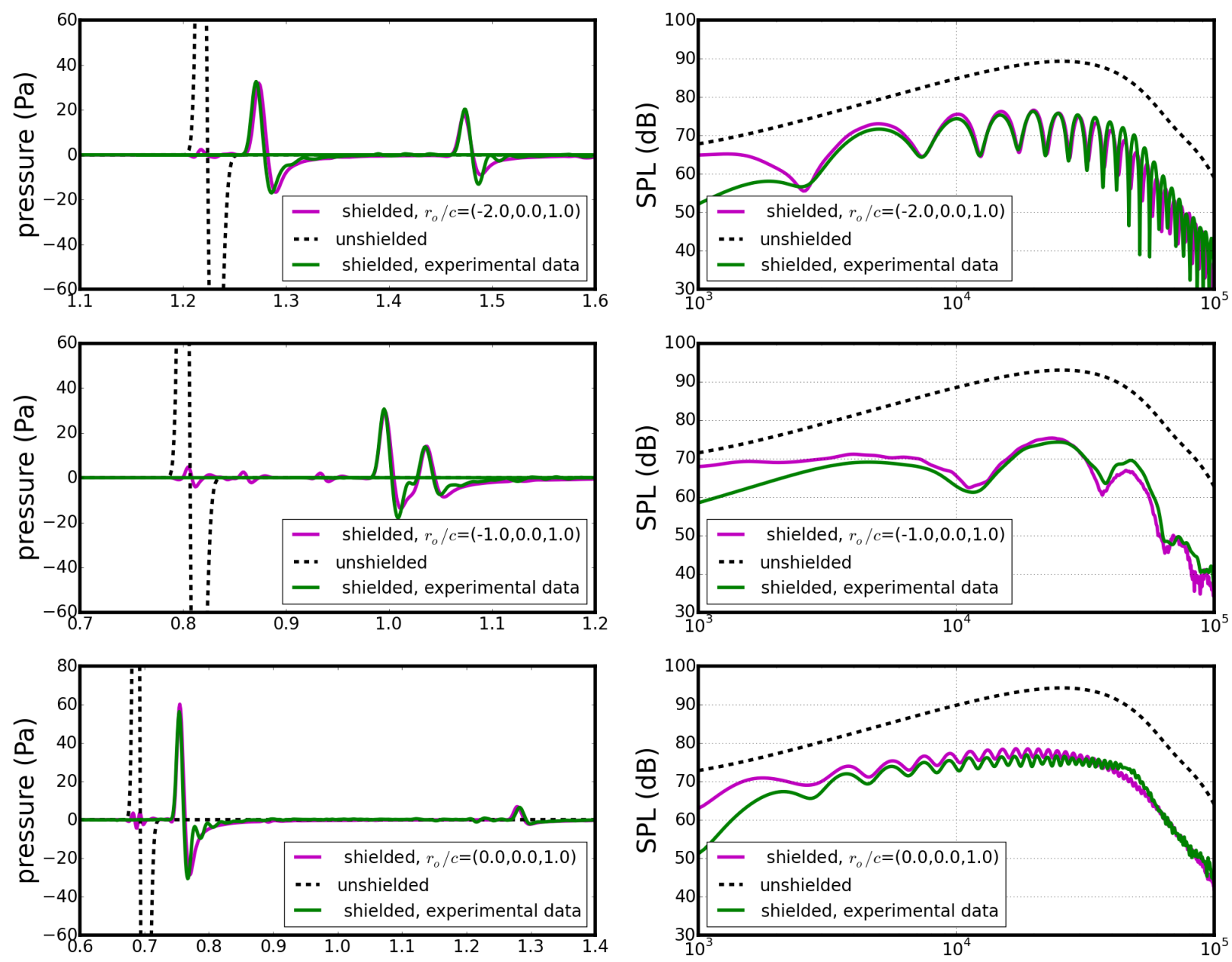

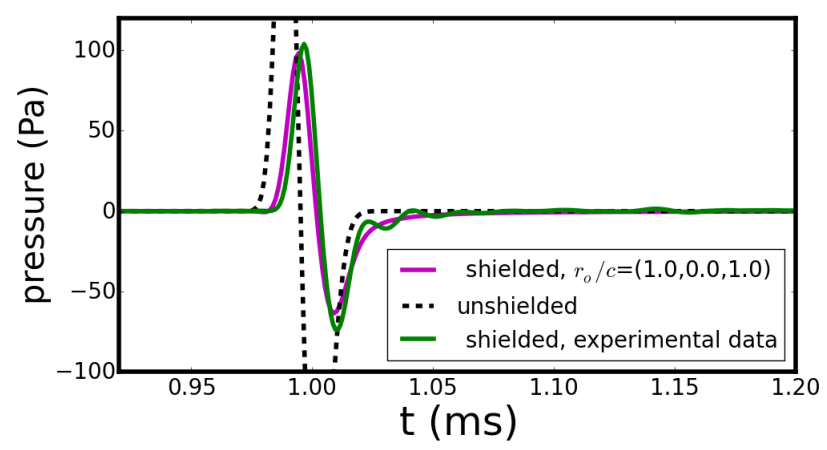

(a)

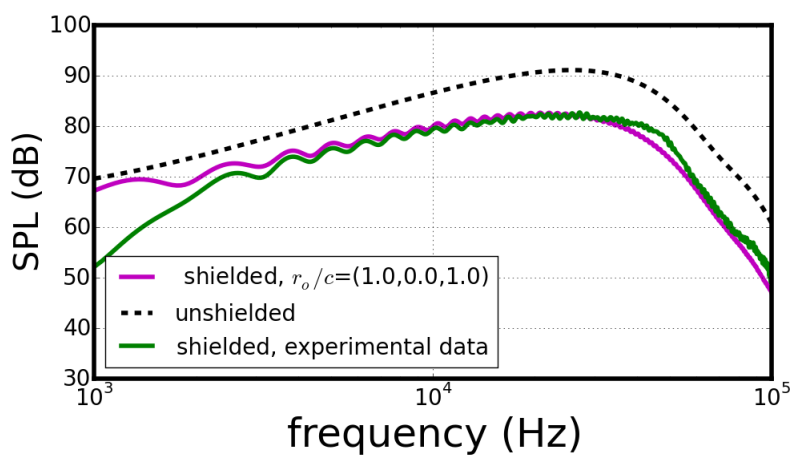

(b)

Figure 23: Comparisons between the computed and measure acoustic pressure at four far field locations as indicated. The source waveform is that of Eq. 15 . The source point is located at $75 \%$ chord at $r_{q}=(-0.25 c, 0,-0.157 c)$. Purple lines are the computation and green lines are the measurements. Dotted lines indicate unshielded direct sound from the point source. (a) time domain; (b) SPL spectrum. 


\section{Acknowledgments}

This research was funded by the Advanced Air Transport Technology (AATT) Project of the NASA Advanced Air Vehicles Program (AAVP). The authors would like to thank Florence Hutcheson for her assistance in obtaining and understanding the QFF experimental data. The authors would also like to thank Karl-Stéphane Rossignol for permission to use data from the joint NACA 0012 sound shielding study.

\section{References}

${ }^{1} \mathrm{Hu}$, F. Q., "An efficient solution of time domain boundary integral equations for acoustic scattering and its acceleration by Graphics Processing Units," AIAA Paper 2013-2018, 2013.

${ }^{2}$ Hu, F. Q., Pizzo, M. E., and Nark, D. M., "On the use of a Prandtl-Glauert-Lorentz transformation for acoustic scattering by rigid bodies with a uniform flow," Journal of Sound and Vibration, Vol. 443, 2019, pp. 198-211.

${ }^{3}$ Hutcheson, F. V., Bahr, C. J., Thomas, R. H., and Stead, D. J., "Experimental Study of Noise Shielding by a NACA 0012 Airfoil," AIAA Paper 2018-2821, 2018.

${ }^{4}$ Rossignol, K.-S., Delfs, J. W., Mößner, M., Gély, D., Bulté, J., and Hutcheson, F., "Experimental Investigations on Noise Shielding: Dependency on Reference Noise Source and Testing Environment," AIAA Paper 2018-2820, 2018.

${ }^{5} \mathrm{Hu}$, F. Q., Pizzo, M. E., and Nark, D. M., "On a time domain boundary integral equation formulation for acoustic scattering by rigid bodies in uniform mean flow," Journal of the Acoustical Society of America, Vol. 142, No. 6, Dec. 2017, pp. 3624-3636.

${ }^{6}$ Hu, F. Q., "Further Development of a Time Domain Boundary Integral Equation Method for Aeroacoustic Scattering Computations," AIAA Paper 2014-3194, 2014.

${ }^{7}$ Hu, F. Q., Pizzo, M. E., and Nark, D. M., "On the Assessment of Acoustic Scattering and Shielding by Time Domain Boundary Integral Equation Solutions," AIAA Paper 2016-2779, 2016

${ }^{8}$ Bahr, C. J., Hutcheson, F. V., and Stead, D. J., "Assessment of Unsteady Propagation Characteristics and Corrections in Aeroacoustic Wind Tunnels Using an Acoustic Pulse," AIAA Paper 2018-3118, 2018.

${ }^{9} \mathrm{Hu}$, F. Q., "On hybrid temporal basis functions for stable numerical solution of time domain boundary integral equations," Advances in Aerodynamics, Vol. 1:9, 2019. 